\title{
CHOOSE: Towards a Metamodel for Enterprise Architecture in Small and Medium-Sized Enterprises
}

\author{
Maxime Bernaert ${ }^{1,2} \cdot$ Geert Poels $^{1} \cdot$ Monique Snoeck $^{3} \bullet$ Manu De Backer ${ }^{1,4}$ \\ ${ }^{1}$ Department of Business Informatics and Operations Management, \\ Ghent University, Tweekerkenstraat 2, B-9000 Ghent, Belgium \\ \{Maxime.Bernaert,Geert.Poels,Manu.DeBacker\}@UGent.be \\ ${ }^{2}$ Master of ICT Enterprise Architecture, \\ inno.com institute, Heiststeenweg 131, B-2580 Beerzel, Belgium \\ Maxime.Bernaert@inno.com \\ ${ }^{3}$ Department of Decision Sciences and Information Management, \\ K.U. Leuven, Naamsestraat 69, B-3000 Leuven, Belgium \\ Monique.Snoeck@KULeuven.be \\ ${ }^{4}$ Department of Management Information Systems, \\ University of Antwerp, Prinsstraat 13, B-2000 Antwerp, Belgium \\ Manu.DeBacker@ua.ac.be
}

\begin{abstract}
Enterprise architecture (EA) is a coherent whole of principles, methods, and models that are used in the design and realization of an enterprise's organizational structure, business processes, information systems, and IT infrastructure. Recent research indicates the need for EA in small and medium-sized enterprises (SMEs), important drivers of the economy, as they struggle with problems related to a lack of structure and overview of their business. However, existing EA frameworks are perceived as too complex and, to date, none of the EA approaches are sufficiently adapted to the SME context. Therefore, this paper presents the CHOOSE metamodel for EA in SMEs that was developed and evaluated through action research in an SME and further refined and validated through case study research in five other SMEs. This metamodel is based on the essential dimensions of EA frameworks and is kept simple so that it may be applied in an SME context. The final CHOOSE metamodel includes only four essential concepts (i.e. goal, actor, operation, object), one for each most frequently used EA focus. As an example, an extract is included from the specific model that was created for the SME used in our action research. Finally, the CHOOSE metamodel is evaluated according to the dimensions essential in EA and the requirements for EA in an SME context.
\end{abstract}

Keywords Enterprise architecture $\bullet$ Small and medium-sized enterprises $\bullet$ CHOOSE $\bullet$ Metamodel

\section{Introduction}

According to IEEE Computer Society (2000), architecture is "the fundamental organization of a system, embodied in its components, their relationships to each other and the environment, and the principles governing its design and evolution". Architecture could thus be defined as "structure with a vision", providing an integrated view of the system designed or studied. At the level of an entire organization, it is commonly referred to as enterprise architecture (EA). This refers to a coherent whole of principles, methods, and models that are used in the design and realization of an enterprise's organizational structure, business processes, information systems, and IT infrastructure (Lankhorst 2013). Rather than specific solutions for specific problems, EA is assumed to capture the essence of the business, IT, and its evolution, as this essence is much more stable. In this respect, EA considers an enterprise as a system in which competencies, capabilities, 
knowledge, and assets are purposefully combined to achieve stakeholder goals. The tangible outcome of this line of reasoning is a blueprint or holistic overview of the enterprise in the form of an integrated collection of models. Hence, architecture can help maintain the essence of the business, while still allowing for optimal flexibility and adaptability (Jonkers et al. 2006).

EA approaches are often experienced as complex, over-engineered, and difficult to implement. Because of the technical detail required for full-scale implementation, EA models tend to become very large, making them more difficult to understand and less effective to reflect on or design enterprises and their supporting systems (Balabko and Wegmann 2006). Due to their resource poverty, SMEs experience even more difficulties than larger enterprises in employing EA experts or hiring external consultants (Kroon et al. 2012). Yet, as some studies have confirmed, they may encounter several problems if they fail to implement EA (Bidan et al. 2012; Bhagwat and Sharma 2007).

Bernaert et al. (2013b) did an extensive problem analysis of EA and SMEs and proposed the concept of EA as a good solution to be used for SMEs to solve problems related to a lack of structure and overview. However, EA is still unknown and hardly used in SMEs. A recent exploratory field study by Bernaert et al. (2013b) examined 27 SMEs and observed that nearly all of them were missing a clear overview of their business organization and none of them actually were using EA (Bernaert et al. 2013b). The authors concluded that there is a pressing need to develop an EA approach specifically adapted to the SME context, consisting of a metamodel, a method, and software tool support.

The goal of the current research is to develop such an EA approach for SMEs, called CHOOSE. As some research has already focused on how to bring EA to SMEs in general (Bernaert et al. 2013b; Bidan et al. 2012; Wißotzki and Sonnenberger 2012; Aarabi et al. 2011; Bernaert and Poels 2011; Jacobs et al. 2011), the value of the current research lies in the fact that, to our knowledge, CHOOSE is the first effort to actually develop an EA approach specifically adapted to the SME context. The present paper will elaborate on the design of the CHOOSE metamodel. The development of the other CHOOSE artefacts is on-going research, consisting of a method to guide the development of CHOOSE models through the instantiation of the metamodel and a suite of software tools to support this instantiation process.

The development of the metamodel was guided by the requirements for EA in an SME context proposed by Bernaert et al. (2013b) and involves a constant trade-off between comprehensiveness and simplicity. Intended for EA, the metamodel needs to provide a holistic overview and thus incorporate the essential dimensions of existing EA approaches. At the same time, though, the metamodel is also intended for SMEs, so it is kept as simple as possible, without being too simple. In order to find the right balance, a set of EA frameworks used in business and academia was analyzed to capture the essential dimensions of EA approaches.

After the essential dimensions of EA approaches had been defined, a suitable starting point for designing the CHOOSE metamodel needed to be found. From different investigated metamodels, the metamodel of the KAOS requirements engineering methodology (Van Lamsweerde 2009) was found to be the most suitable as it is rather elaborate and provided a good match with the essential dimensions that had been determined. 
Next, during multiple rounds of action research (Järvinen 2007) in one specific SME that complied with the characteristics of SMEs as proposed by Bernaert et al. (2013b), the KAOS metamodel was adapted and transformed into the CHOOSE metamodel. Some of the changes to the developing metamodel were, however, triggered by parallel case study research in five other SMEs, which was initiated to design the CHOOSE method. Any changes that the action research participants considered useful were also incorporated into the final CHOOSE metamodel.

This final metamodel comprises four viewpoints: (1) a goal viewpoint for the motivational part (i.e. why), (2) an actor viewpoint for the active performers (i.e. who), (3) an operation viewpoint for the behavioural part (i.e. how), and (4) an object viewpoint for the description of the concepts and relationships (i.e. what). In this way, the core part of the CHOOSE metamodel only consists of the bare minimum of concepts (only one main concept per viewpoint) in order to maintain the balance between both comprehensiveness for EA and simplicity for SMEs. Since in the original KAOS metamodel all the viewpoints are tightly integrated, in the resulting CHOOSE metamodel also a high traceability within and between the four viewpoints was achieved.

The CHOOSE metamodel is written in UML (Unified Modeling Language). Its elements are defined using SBVR (Semantics of Business Vocabulary and Rules) and intra- and inter-view constraints are specified as OCL (Object Constraint Language) constraints. These SBVR definitions are based on definitions of well-known modelling languages and thus contribute to the unambiguous definition of the metamodel concepts. The OCL constraints, in their turn, help ensure the completeness and consistency of the models that instantiate the metamodel.

The instantiation of the proposed metamodel is demonstrated by means of the EA model that was developed during the action research programme in the SME. This also provided the basis for the evaluation of the metamodel, a process that was guided by the EA essential dimensions and the requirements for EA in SMEs.

Section 2 of this paper elaborates on the research problem, the intended contribution of this research, and the requirements for EA in an SME context. In section 3, the solution approach, the scope of the research presented in this paper, and the research methodology are presented. The results are shown from section 4 onwards: the definition of essential EA dimensions based on an analysis of EA frameworks (section 4); the choice of KAOS as a starting point for the metamodel design (section 5); the adaption of the initial metamodel and the development of the CHOOSE metamodel during the action research and case studies (section 6); the formal definition of the resulting CHOOSE metamodel (section 7); and, finally, its evaluation (section 8). The final section, section 9, presents conclusions and outlines the current and future research required to complete the development of CHOOSE.

\section{Problem Description and Solution Requirements}

This section describes the research problem and the requirements for its solution, based on a review of related and previous research.

\subsection{Problem Description}

A good EA gives a static overview of the enterprise and offers a means for supporting change. A good architectural practice helps a company innovate and change, by providing both stability and 
flexibility (Jonkers et al. 2006). Jonkers et al. (2006) further mention that it is important to realize that most stakeholders of a system are probably not interested in its architecture, but only in the impact of this architecture on their concerns. In addition, although they often have radically different backgrounds, an architect should be able to explain the architecture to all of the stakeholders just as clearly. This highlights one of the most important roles of EA: it serves as an instrument in the communication among diverse groups and interests and produces a common ground for discussion and decision-making.

EA has become one of the top priorities of IT executives and is considered an important instrument for aligning the required changes in corporate strategy and business processes with an increasingly complex IT landscape (Luftman and Ben-Zvi 2011). Some of the most recognized benefits of EA are that IT can be used more efficiently and flexibly, business and IT can be better aligned (Radeke 2011; Tamm et al. 2011; Daneva and van Eck 2007; Lindström et al. 2006), and a better fit between business operations and strategy can be achieved (Hoogervorst 2004; Veasey 2001). Braun and Winter (2005) underscore that in order for business-IT and strategy to be aligned, EA must be adaptable and constantly held up-to-date.

SMEs constitute over $90 \%$ of operating businesses in many countries, in the U.S. even $99.7 \%$ (Small Business Administration 2011) and in Europe 99.8 \% (European Commission 2011). There is therefore a great need for more rigorous research that is relevant for this important sector of the economy (Devos 2011).

Right now, existing EA frameworks are primarily used in large enterprises (Gartner 2012). Wißotzki and Sonnenberger (2012), among others, recognize the importance of EA and EA management (EAM) in particular, but also notice that EAM is still mostly unexplored and rarely used, especially in the context of SMEs (see also (Bernaert et al. 2013b; Devos 2011). Yet, such specific research is crucial, as research findings based on large businesses cannot be generalized to small businesses due to the inherent differences between SMEs and large businesses (Aarabi et al. 2011).

Lybaert (1998) discovered that SME owners or managers with a greater strategic awareness use more information and that SMEs that use more information are generally more successful. Hannon and Atherton (1998) further revealed that for SMEs success is correlated with higher levels of strategic awareness and better planning of owners-managers. In addition, there is evidence to believe that companies that make strategic rather than just financial business plans perform significantly better financially than those that do not (O'Regan and Ghobadian 2004; Smith 1998). Jacobs et al. (2011) argue that from the perspective of change and complexity, EA could assist SME management during the growth of a small enterprise. For example, according to Aarabi et al. (2011), ERP (Enterprise Resource Planning) systems cannot be successfully implemented and utilized in SMEs if EA is disregarded. In fact, it is EA's integration of strategic goals, business processes, and technology planning methods that provides the standards, roadmap, and context for ERP implementation (Zach 2012). As Bidan et al. (2012) conclude, process standardization in SMEs is more important than the deployment of technology (e.g., ERP systems) to improve organizational performance. In short, SMEs need to get a structured view of their company, even before they start implementing an ERP solution. 
Hence, while EA might offer SMEs a solution to typical problems related to a lack of overview, strategic awareness, IT planning, and business-IT alignment, EA approaches that cater for the specificities of small businesses are still missing. This lack of research on an EA approach that can readily be used for SMEs is exactly the problem that is addressed in the present research.

\subsection{Requirements for EA for SMEs}

To guide the development and evaluation of an EA approach for SMEs, requirements for an appropriate solution are needed. These requirements were specified in previous research (Bernaert et al. 2013b) and will be summarized here. First, the requirements for EA in general are presented, followed by those for the adoption and successful use of IT in SMEs. To end, the combination of these two sets of requirements into a single set for EA in an SME context, as per (Bernaert et al. 2013b), is also described.

\subsubsection{Requirements for $E A$}

The essential requirements for EA (Bernaert et al. 2013b; Lankhorst 2013; Zachman 1987) are the following:

1. Control: EA should be usable as an instrument in controlling the complexity of the enterprise and its processes and systems.

2. Holistic Overview: EA should provide a holistic overview of the enterprise and be able to capture its essence: the stable elements that do not vary across specific solutions found for the problems currently at hand.

3. Objectives: EA should facilitate the translation from corporate strategy into daily operations.

4. Suitability: EA should be suitable for its target audience. It needs to be understood by all those involved, even if they come from different domains.

5. Enterprise-wide: EA should enable optimization of the company as a whole instead of doing local optimization within individual domains.

The fourth requirement refers to the target audience. In our case, the target audience is SMEs and, more specifically, their owners or managers. Therefore, requirement 4 is refined using the requirements for the adoption and successful use of IT in SMEs. This topic has been dealt with extensively in several studies, listed by Bernaert et al. (2013b). The authors argue that since Moody (2003) showed that IT adoption models are also useful for evaluating the adoption of ITrelated methods (e.g., information systems design methods), and that EA, with its origins in IT research (Zachman 1987), can be seen as such a method, IT adoption models for SMEs can provide useful insight into the determining factors for successfully using EA in SMEs.

\subsubsection{Requirements for the Adoption and Successful Use of IT in SMEs}

The requirements for the adoption and successful use of IT in SMEs (Bernaert et al. 2013b) are as follows:

4.1 The approach should enable SMEs to work in a time-efficient manner on strategic issues.

4.2 A person with limited IT skills should be able to apply it.

4.3 It should be possible to apply the approach with little assistance of external experts.

4.4 The approach should enable making descriptions of the processes in the company. 
4.5 The CEO must be involved.

4.6 The expected revenues of the approach must exceed the expected costs and risks.

By combining these requirements with the EA requirements of the previous section, Bernaert et al. (2013b) obtained a set of requirements for the adoption and successful use of EA in SMEs.

According to requirement 4 and thus 4.1-4.6, the EA model should be understandable and adaptable by non-EA experts in SMEs. The previously mentioned role of EA as a communication instrument can only be established by tailoring an EA approach to the specificities of SMEs. Bernaert et al. (2013b) therefore argue for a different EA approach for SMEs, based on simplicity. We are fully aware that focusing on simplicity rather than on completeness is not common in an academic context. However, also Balabko and Wegmann (2006) emphasized that current EA approaches are often experienced as complex, over-engineered, and difficult to implement.

\section{Solution Approach and Research Methodology}

In this section, we will present CHOOSE as the solution to the problem described in the previous section. We will limit the scope of the research presented in this paper to the primary artefact of CHOOSE (i.e. its metamodel) and we will describe the research methodology that was followed to develop and evaluate this metamodel.

\subsection{CHOOSE: Balancing Comprehensiveness and Simplicity}

Our solution consists of developing a new EA approach guided by the requirements for EA in an SME context (cf. section 2.2). The approach was called CHOOSE, so that these requirements would always be kept in mind. CHOOSE is an acronym for "maintain Control, by means of a Holistic Overview, that is based on Objectives and kept Simple, of your Enterprise".

It is clear that the development of the CHOOSE metamodel will involve an on-going assessment of comprehensiveness and simplicity (see the methodological pragmatism (Rescher 1977)), because it should include the necessary information to get a holistic overview of the enterprise, while still being as simple as possible. As Albert Einstein once said, "A scientific theory should be as simple as possible, but no simpler".

The meaning of simplicity and complexity of a metamodel can be found in related work by Erickson and Siau (2007), in which a simplified core of the UML metamodel is proposed, based on key constructs. They argue that any increase of this core comes at the expense of increased complexity. Their work is mainly based on the work of Rossi and Brinkkemper (1996), who argued that "the relative complexity of methods and techniques based on metamodels is significant because it can be expected to affect the learnability and ease of use of a method". In other words, the number of metamodel objects, relationships, and properties to be learned adds to the complexity.

There is of course a trade-off between a metamodel's learnability and its expressive power. When organizations select metamodels, they should be aware that more powerful metamodels may be harder to learn, yet may also be more effective for experienced users. As previously mentioned, though, related research on EA in SMEs shows that SMEs hardly use EA, even hardly know about its existence, and can therefore be seen as novice users. 


\subsection{Research Process and Scope}

This work extends the earlier research by Bernaert et al. (2013b). Their research investigates why EA has not yet been adopted by SMEs, despite its possible benefits. In this respect, Bernaert et al. (2013b) also present a research process (Fig. 1) for developing an EA approach adapted to the SME context.

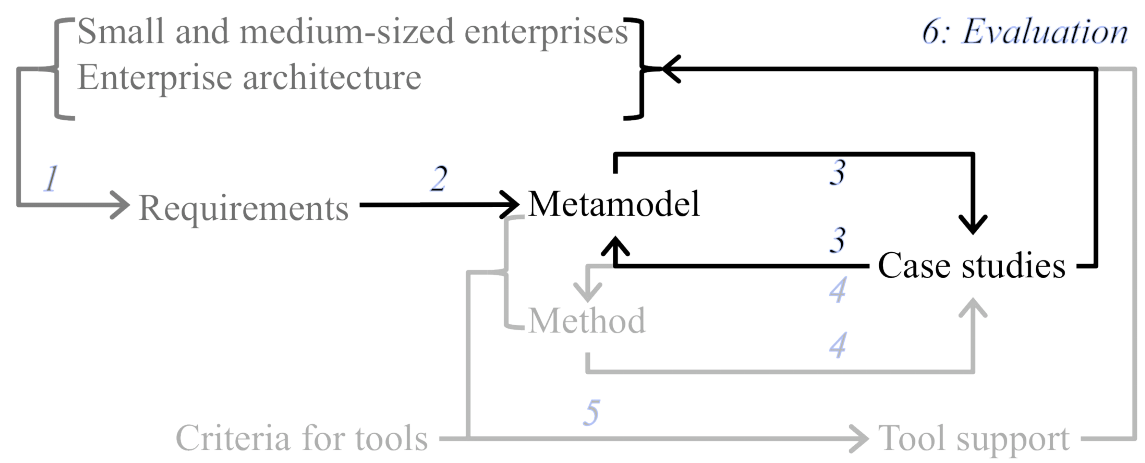

Fig. 1 Research process for developing an EA approach for SMEs (from (Bernaert et al. 2013b))

The dark grey lines in Fig. 1 express the work that has been done by (Bernaert et al. 2013b). In step 1, both the literature on EA and IT use in SMEs were analyzed and relevant characteristics were examined. From these characteristics, requirements were extracted for EA in an SME context, which have already been summarized in this paper in section 2.2 .

The black lines in Fig. 1 highlight the part of the research process that is reported in this paper. The light grey lines in Fig. 1 refer to the (on-going) research required for developing the CHOOSE method and supporting software tools, which lies beyond the scope of this paper.

Step 2 was desk research based on a literature study and analysis, which involved choosing a suitable starting point to design the CHOOSE metamodel. While constantly keeping in mind the balance between comprehensiveness and simplicity, we analyzed a large number of existing EA frameworks in order to extract the essential dimensions of EA frameworks. In the end, an initial metamodel (i.e. the KAOS metamodel) was selected that matched these dimensions.

Step 3 was field research conducted primarily by means of action research in an SME and complemented with case study research in five other SMEs. Through the action research programme, the metamodel was gradually further developed, with the initial metamodel as a starting point. The outcome of the action research was also used to evaluate the research results with respect to the EA essentials and the requirements for EA in an SME context (step 6).

After the start of the action research, five case studies involving the use of CHOOSE were initiated in SMEs with different characteristics (e.g., size, sector). These case studies were primarily used to develop the CHOOSE method (step 4). As the development of this method required us to implement CHOOSE, the initial version of the metamodel that was available at that time in the action research programme was also tested in these other SMEs. Hence changes to the initial metamodel were also tested in other SMEs. Conversely, the experiences in the case study companies were used as additional input to the action research. Therefore, when necessary, these other case studies are briefly referred to in section 6 , where the development of the CHOOSE metamodel is described. 


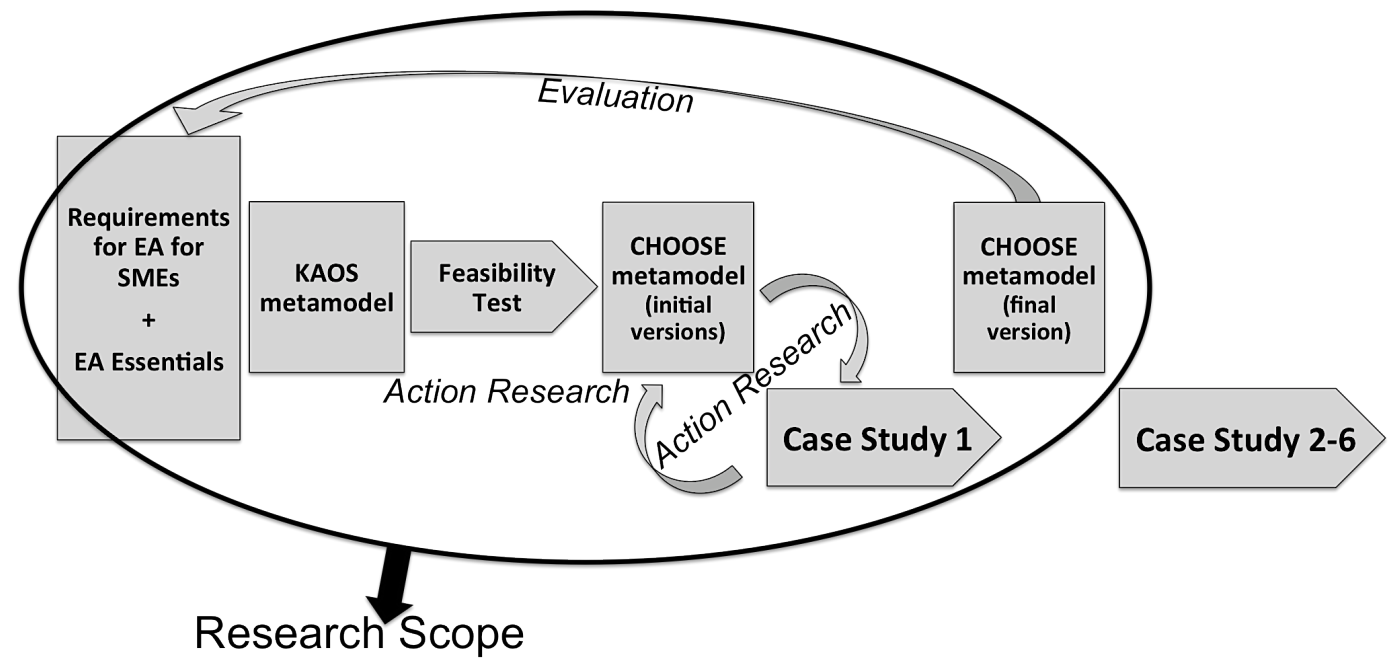

Fig. 2 Research process for developing the CHOOSE metamodel

\subsection{Action Research}

The main research methodology employed in step 3 of Fig. 1 was action research (Susman and Evered 1978). Action research employs the researcher as an active participant rather than a passive observer. It is a cyclical process of actively participating in an enterprise change situation while at the same time doing research. The basic steps are planning (i.e. problem identification), acting (i.e. changing and learning processes), and evaluating (i.e. measuring results) (French and Bell 1973). According to Järvinen (2007), action research is an instance of the design science methodology (Hevner et al. 2004) that is suitable when little theoretical background or experience is available, which is the case for the implementation of EA in SMEs.

Baskerville and Myers (2004) provide three guidelines for good action research, which we applied as follows:

1. Demonstrate a contribution or potential contribution to practice (i.e. the action): EA for SMEs could provide SMEs with solutions to problems related to a lack of structure and overview (Bernaert et al. 2013b).

2. Demonstrate a clear contribution to research (i.e. the theory): This research develops the CHOOSE metamodel for EA in an SME context, an artefact that can be further refined and tested in other research.

3. Identify the criteria by which to judge the research and demonstrate how these criteria are met: The criteria for our research were presented as requirements for EA in an SME context in section 2.2 and are part of the evaluation in section 8 .

The action research was performed in multiple rounds in an SME that sells car tyres and performs small maintenance jobs on cars (i.e. case study 1 in Fig. 2). It has six permanent employees and works with temporary employees during the busy winter season. This SME was chosen because it complied with the common characteristics of SMEs (Bernaert et al. 2013b): management has little time to look at strategic matters, no EA experts are employed, no funds to hire external consultants are available, the extent of employees' responsibility for certain tasks is often discussed, the CEO is the central figure, and the CEO takes the decision of whether or not to adopt a new approach. 
In the first action research cycle, of which the results were published in (Bernaert and Poels 2011), the KAOS metamodel was used in its original form as a feasibility test (see Fig. 2) to see if it could be used to model the EA of an SME. It turned out that KAOS in fact did have the ability to document and analyze the EA of an SME, although it was originally developed for modelling software-intensive systems within their organizational or physical environment (Van Lamsweerde 2009). Nevertheless, the test also showed that the metamodel needed to be adjusted in order to change its scope from a system on the software level (KAOS) to a system on the enterprise level (CHOOSE). This called for more action research cycles.

Four further cycles of action research were performed (see action research cycles in Fig. 2). In each round, the CEO of the SME was involved in completing the SME's EA model according to the CHOOSE metamodel version available at that moment. To ensure more objectivity in evaluating the results, in each round two researchers were involved to obtain investigator triangulation (Denzin 2006). Each round was voice recorded to obtain raw data and both researchers made additional notes. The voice recordings, notes, and models were stored in a case study database. As most of the data involved strategic issues, a limitation of this research is that the case study database contains confidential data and cannot be made public.

To analyze the data obtained in each action research cycle, the process presented in (Susman and Evered 1978) was followed:

- Diagnosing: The model, voice recordings and the notes of both researchers were analyzed, on the basis of which a list was established of encountered problems that called for adaptations to the metamodel.

- Action planning: For each problem, a set of possible adaptations to the metamodel was considered by the researchers, favouring adaptations that were likely to be more generally accepted by CEOs of SMEs.

- Action taking: The SME's EA model was changed according to the proposed adaptations to the metamodel.

- Evaluating: The model changes were evaluated to see if the problems were solved and if new problems would surface.

- Specifying learning: Positively evaluated adaptations were included in the next version of the CHOOSE metamodel.

As expected, after each round fewer changes had to be made and after three of the four additional rounds the metamodel had become stable. In the meantime, some other adaptations triggered by the case study research in the other five SMEs (i.e. case studies 2-6 in Fig. 2) were tested and evaluated in the SME used in the action research. If these adaptations were positively evaluated, they too became part of the final version of the CHOOSE metamodel.

This final metamodel provided input for the development of prototype software tools (step 5 in Fig. 1) (Bernaert et al. 2013a; Dumeez et al. 2013; Ingelbeen et al. 2013). One such tool was installed in the SME in the last round and enabled it to manage its EA model after the end of the action research programme. As such, this tool can supply longer-term feedback on the CHOOSE metamodel. 


\section{Essential Dimensions of Enterprise Architecture}

In this section, existing EA frameworks are first reviewed, so that the most important ones may be pinpointed. Next, the identified frameworks are analyzed in order to determine the essential EA dimensions. These dimensions are then used in the next section to help select a suitable starting point to design the CHOOSE metamodel.

\subsection{Enterprise Architecture Frameworks}

Since the publication of the Zachman framework in 1987 (Zachman 1987), a multitude of EA frameworks have been proposed. In order to identify the essential elements of an EA metamodel in its most simple form, balancing comprehensiveness and simplicity (see section 3.1 ) and meeting the EA requirements for SMEs (see section 2.2), this section aims to identify the most common elements in the most important EA frameworks proposed so far. These essential dimensions of EA define the degree of freedom that can be exerted in adapting the CHOOSE metamodel during the action research cycles, as they set clear and minimal boundaries for the key elements that the metamodel should include.

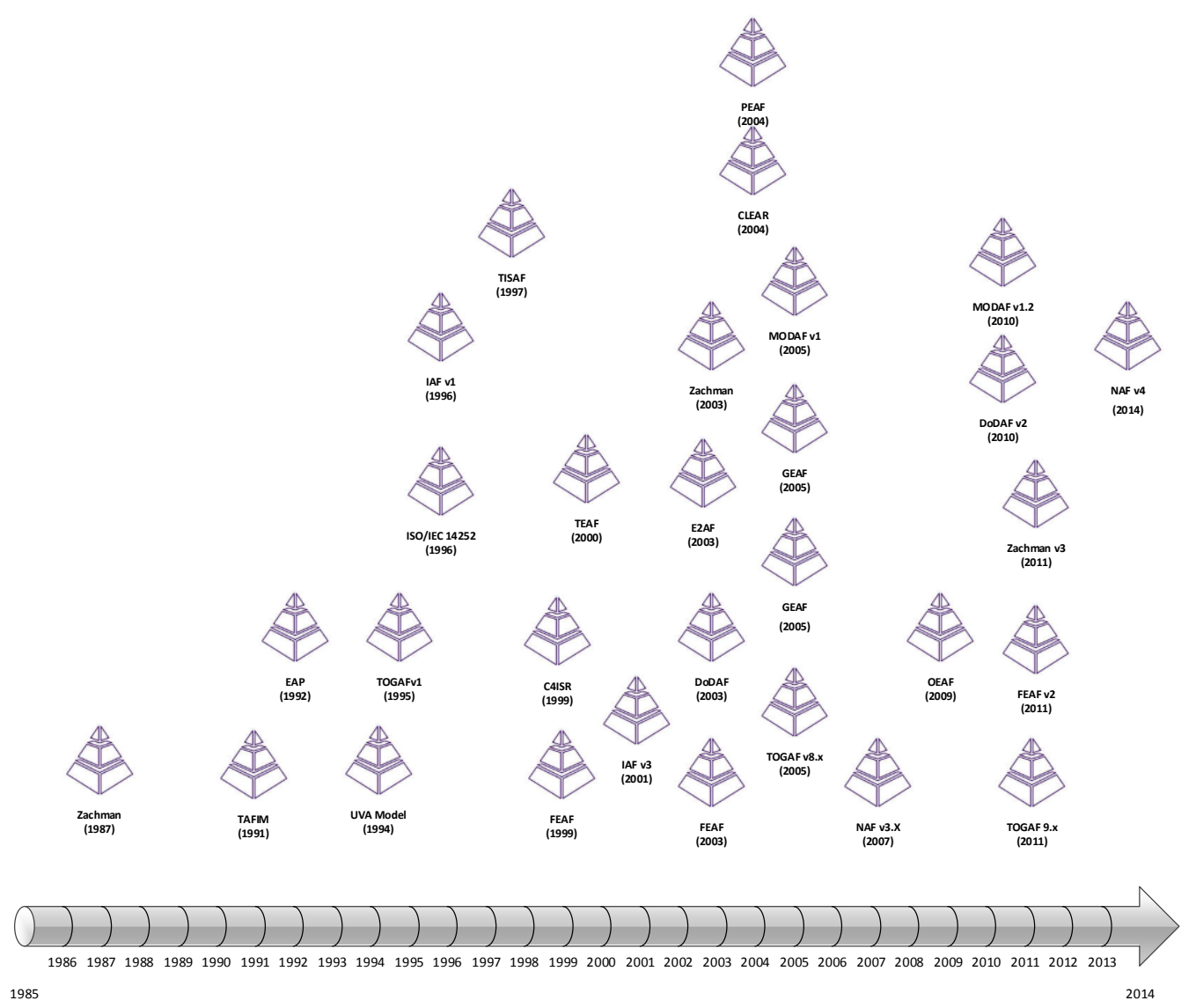

Fig. 3 Historical overview of EA frameworks (updated by Georgadis (2015) from (Schekkerman 2006))

To identify the most important frameworks, we studied several reviews and historical overviews of EA frameworks, such as the one provided by Georgiadis (2015) (Fig. 3). The overview by Schekkerman (2006) is less recent, but interesting for its explanation of the influences EA frameworks have had on each other. Based on these influence relationships, Zachman (Zachman International 2011), TOGAF (The Open Group 2009), DoDAF (DoD 2010), and E2AF 
(IFEAD 2006) appear to be important EA frameworks. Zachman gave rise to another EA framework, TEAF, which was created for the US Department of the Treasury. Yet, since it is subsumed in the Federal Enterprise Architecture (FEA), just like FEAF, it is better to include FEA instead of TEAF.

Zachman, TOGAF, DoDAF, FEAF, and TEAF are all analyzed in the study of Urbaczewski and Mrdalj (2006). Sessions (2007), on the other hand, compares the first two, Zachman and TOGAF, with FEA and Gartner's GEAM. Yet another study by Leist and Zellner (2006) juxtaposes Zachman, TOGAF, DoDAF, FEAF, TEAF, ARIS, and MDA (model-driven architecture). The last one, MDA, is more a general systems development approach, so it will not be included in our further analysis here.

In short, the most widely discussed EA frameworks that should also be included in the present analysis are Zachman (Zachman International 2011), TOGAF (The Open Group 2009), DoDAF (DoD 2010), E2AF (IFEAD 2006), FEA (The White House OMB 2013, 2012), GEAM (Gartner) (Bittler and Kreizmann 2005; James et al. 2005), and ARIS (Scheer 2000) (see Table 1).

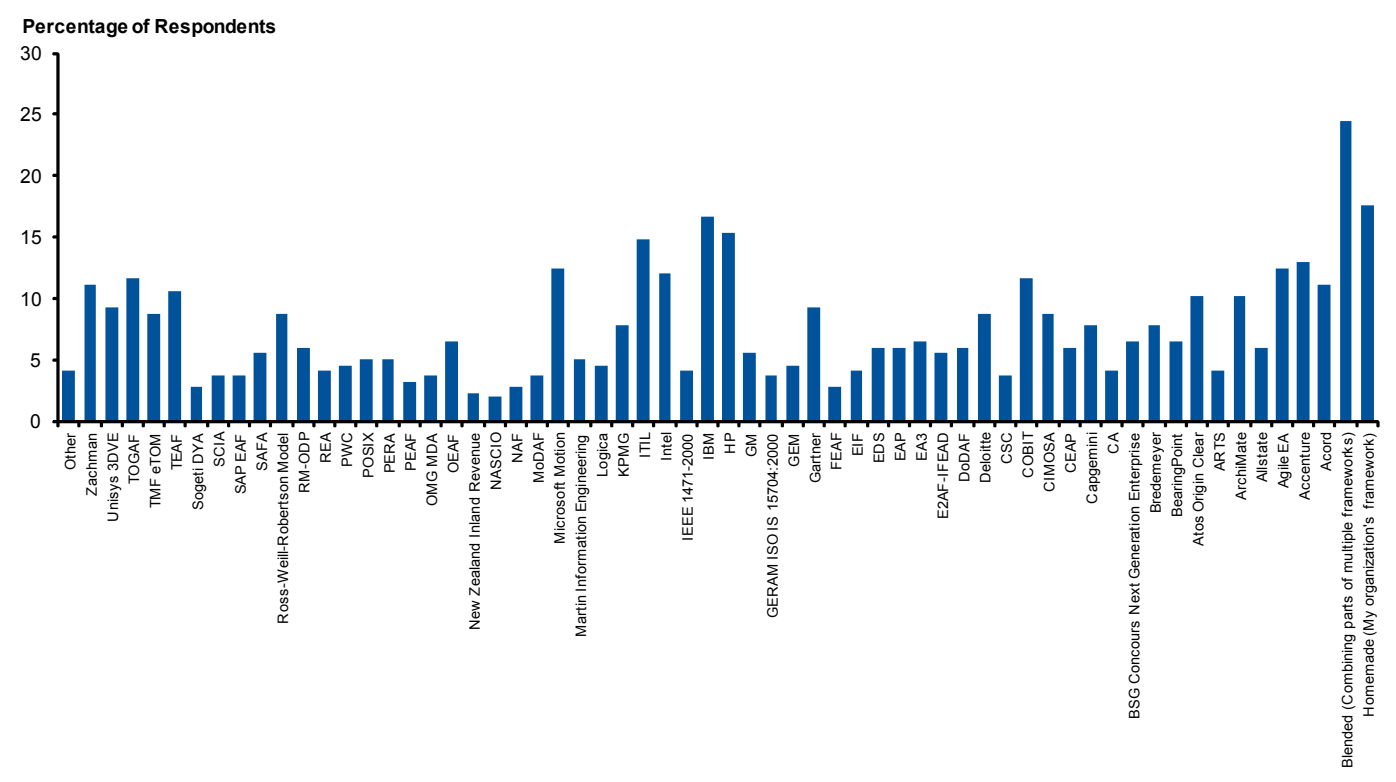

$\mathrm{N}=216$

Fig. 4 EA frameworks currently being used (from (Gartner 2012))

This selection of relevant EA frameworks is confirmed by the survey of IFEAD (2005) and, more recently, by the survey of Gartner (2012) on the use of EA frameworks in companies (Fig. 4). However, a lot of companies also use a homemade EA framework or hire a consulting firm (e.g., IBM, Deloitte) to help them craft a best-of-breed framework. ArchiMate (Lankhorst 2013) was also included in Table 1, because it was recently adopted as a standard by The Open Group (2012) to be used in combination with TOGAF. Capgemini's IAF (van 't Wout et al. 2010) was also added because it was built based on experience in more than 3000 EA projects and it evolves faster than any standard ever can. As such, it lies at the basis of large parts of TOGAF 9's content framework. The Business Motivation Model (BMM) (OMG 2010) is also relevant for our study because of its emphasis on the motivational dimension. Yet it does not give a holistic EA overview and is not actually an EA framework, so it has been placed between brackets in Table 1. At the same time, though, BMM is often included in business architecture analyses (Glissman and Sanz 
2009), so it should definitely be taken into account in our analysis. Finally, Sogeti's DYA (Wagter et al. 2005) offers a holistic view and should therefore also be included in Table 1.

To make sure that recently developed EA frameworks were not ignored, we also included several EA frameworks developed in academia, namely CARP (derived from DoDAF) (Business Transformation Agency 2009), Enterprise Modelling (Bubenko 1993) and its successors Enterprise Knowledge Development (EKD) (Stirna and Persson 2007) and 4EM (Sandkuhl et al. 2014), REA extended with goal modelling (Andersson et al. 2009) (Fig. 4), SEAM (Wegmann et al. 2007), and LEAP (Clark et al. 2011).

\subsection{Essential Dimensions of EA}

The essential dimensions of EA were determined in three consecutive steps.

Firstly, according to Schekkerman's (2006) and Georgiadis' (2015) overview of EA influence history, Zachman (1987) seems to be at the very origin of many EA frameworks. The collection of EA frameworks identified in the previous section will therefore be analyzed by means of the six focuses (columns) of the Zachman framework (what, how, where, who, when, why). These focuses make it possible to classify architectural descriptions according to content or subject focus (e.g., objects or data for what, processes for how, networks or locations for where, etc.), so that architecture models according to a particular focus represent a single aspect of the enterprise, abstracting from relationships with the other aspects.

Secondly, Winter and Fischer (2007) identified five essential architectural layers in EA frameworks (i.e. business, process, integration, software, infrastructure). These architectural layers allow a further classification of (parts of) architectural descriptions, so that architecture models are expressed using concepts that represent the enterprise elements that are relevant to a certain perspective, in a way that is comprehensible for the stakeholders in that perspective. The process architecture layer can then be further merged with the business architecture layer and, in its turn, the integration architecture layer can be combined with the software architecture layer. This results in three essential EA layers: business, software, and infrastructure. These layers were used to analyze the EA frameworks in Table 1 (i.e. business (B), software (IS), infrastructure (IT), or a blend of the three).

Thirdly, during the analysis of the selected EA frameworks, no additional focuses or architectural layers were identified. However, what also became apparent during the analysis was that most EA frameworks make it possible to translate strategy into operations and often stress the importance of a long and thorough analysis of the strategy space, free from all implementation constraints. Lankhorst (2013), for instance, refers to the strategic alignment model of Henderson and Venkatraman (1993), according to which EA can help in executing the business or IT strategy and enable the alignment between (business or IT) strategy and (organizational or IT) infrastructure and processes. In fact, many EA frameworks provide guidance for the translation from corporate strategy into daily operations. For example, Zachman (2011) defines six views (rows) from "scope" all the way to the "full enterprise", adding more implementation constraints towards the "full enterprise" view. Another example is IAF (van 't Wout et al. 2010), which is primarily built upon the principle of analyzing the strategy space for as long as possible without 
taking into account the constraints of operations beforehand, by using contextual, conceptual, logical, and physical abstraction levels that are closely related to the different views of Zachman.

Since this aspect can be found in many EA studies, we too have decided to incorporate this, so the last column of our analysis shows whether the EA frameworks provide a means to analyze the (business or IT) strategy space while still disregarding the constraints of (organizational or IT) operations (i.e. strategy-operations).

Table 1 gives an overview of the analyzed EA frameworks. For each Zachman focus, one or more concepts that represent enterprise elements according to that focus are provided as examples, if they are defined in the metamodel of the EA framework. In the strategy-operations column, a minus/plus-minus/plus indicates that a translation from (business or IT) strategy into (organizational or IT) operations is not/limited/clearly supported.

Table 1 Analysis of EA frameworks

\begin{tabular}{|c|c|c|c|c|c|c|c|c|}
\hline & WHAT & HOW & WHERE & WHO & WHEN & WHY & $\begin{array}{l}\text { BUSINESS } \\
\text { IS } \\
\text { IT }\end{array}$ & $\begin{array}{c}\text { STRATEGY } \\
- \\
\text { OPERATIONS }\end{array}$ \\
\hline Zachman & What & How & Where & Who & When & Why & $\mathrm{B} / \mathrm{IS} / \mathrm{IT}$ & + \\
\hline TOGAF & $\begin{array}{c}\text { Data entity, } \\
\ldots\end{array}$ & Process, ... & $\begin{array}{l}\text { Infrastructure } \\
\text { extension }\end{array}$ & $\begin{array}{l}\text { Organization unit, } \\
\text { actor, role, ... }\end{array}$ & Event, ... & $\begin{array}{l}\text { Motivation } \\
\text { extension }\end{array}$ & $\mathrm{B} / \mathrm{IS} / \mathrm{IT}$ & + \\
\hline ArchiMate & Information & Behaviour & Network, ... & Structure & Event, ... & Motivation & $\mathrm{B} / \mathrm{IS} / \mathrm{IT}$ & + \\
\hline DoDAF & Resource & Activity & Location & Performer & - & Capability & Blend & + \\
\hline CARP & Resource & Activity & - & Performer & - & Capability & B & + \\
\hline IAF & Object & Activity & Interaction & Actor, role, ... & Event & Why, goal, ... & $\mathrm{B} / \mathrm{IS} / \mathrm{IT}$ & + \\
\hline E2AF & $\begin{array}{c}\text { Business } \\
\text { objects, } \\
\text { resources, ... }\end{array}$ & $\begin{array}{c}\text { Business } \\
\text { activities, } \\
\ldots\end{array}$ & With who? & $\begin{array}{c}\text { Organization } \\
\text { structure, actors, } \\
\ldots\end{array}$ & When? & Why? & $\mathrm{B} / \mathrm{IS} / \mathrm{IT}$ & + \\
\hline FEA: FEAF & Objects, ... & $\begin{array}{l}\text { Business } \\
\text { process, ... }\end{array}$ & $\begin{array}{c}\text { Business } \\
\text { locations, ... }\end{array}$ & - & - & - & IS/IT & - \\
\hline FEA: TEAF & $\begin{array}{c}\text { Information, } \\
\ldots\end{array}$ & $\begin{array}{l}\text { Business } \\
\text { process, ... }\end{array}$ & $\begin{array}{l}\text { Information } \\
\text { exchange, ... }\end{array}$ & $\begin{array}{c}\text { Organization } \\
\text { chart, ... }\end{array}$ & Event, ... & $\begin{array}{c}\text { Mission, vision, } \\
\ldots\end{array}$ & $\mathrm{B} / \mathrm{IS} / \mathrm{IT}$ & + \\
\hline GEAM & - & - & - & - & - & $\begin{array}{l}\text { Requirements } \\
\text { vision }\end{array}$ & $\mathrm{B} / \mathrm{IS} / \mathrm{IT}$ & + \\
\hline ARIS & $\begin{array}{c}\text { Input, output, } \\
\ldots\end{array}$ & Function & - & $\begin{array}{c}\text { Organizational } \\
\text { unit, ... }\end{array}$ & Event & Goal & Blend & +- \\
\hline (BMM) & - & $\begin{array}{l}\text { (Business } \\
\text { process) }\end{array}$ & - & $\begin{array}{l}\text { (Organization } \\
\text { unit) }\end{array}$ & - & End & B & + \\
\hline DYA & $\begin{array}{l}\text { Product, } \\
\text { data, ... }\end{array}$ & Process & Network & Organization, ... & - & $\begin{array}{l}\text { Business } \\
\text { objectives }\end{array}$ & $\mathrm{B} / \mathrm{IS} / \mathrm{IT}$ & + \\
\hline $\begin{array}{l}\text { Enterprise } \\
\text { modelling / } \\
\text { EKD / 4EM }\end{array}$ & $\begin{array}{l}\text { Concepts } \\
\text { model }\end{array}$ & $\begin{array}{c}\text { Business } \\
\text { process } \\
\text { model }\end{array}$ & - & $\begin{array}{l}\text { Actors and } \\
\text { resources model }\end{array}$ & - & Goals model & $B / I S$ & + \\
\hline REA & Resource & Event & - & Agent & - & Goals & B & - \\
\hline SEAM & - & - & - & - & - & Strategies & $\mathrm{B} / \mathrm{IS}$ & + \\
\hline LEAP & Object & Operation & - & Object & Condition & OCL constraint & $\mathrm{B} / \mathrm{IS} / \mathrm{IT}$ & - \\
\hline
\end{tabular}

Most of the frameworks use (at least) four focuses from Zachman's framework: what, how, who, why. The where-focus is usually only implicitly present in, for instance, relationships between elements and in networks. Often, an explicit metamodel concept for expressing enterprise elements according to this focus is missing. The when-focus, if used, is mostly related to conditions or events that trigger processes. In this respect, it is closely related to and often included in the how-focus (e.g., event-driven process chains). Yet, Winter and Fischer (2007) argue that in EA, "business processes should not be decomposed further than to the subprocess level. Detailed process descriptions including specifications of activities and work steps are out of EA scope and should be maintained by using specialized business process modelling tools". This holistic overview function of EA is confirmed by other authors, such as (Lankhorst 2013; Jonkers et al. 2006). 
The importance of these four focuses is confirmed by a large number of application cases performed with EKD. Stirna and Persson (2007) point out that, while EKD specifies six submodels, it focuses predominantly on the goals model, business process model, concepts model, and actors and resources model. According to these authors, these sub-models correspond to the why, how, what and who questions, which are the four essential Zachman focuses that we identified. EKD sub-models thus represent a single aspect of the enterprise using concepts related to a particular focus.

Most of the time, all three layers (i.e. business (B), software (IS), infrastructure (IT)), or a blend of them are used. Most EA frameworks also emphasize the importance of analyzing the strategy space without worrying about the constraints of operations beforehand (strategy-operations).

Hence, these three things are defined as the essential EA dimensions to be supported by the CHOOSE metamodel: (1) the presence of the four focuses (why, who, how, what), (2) at least a blend of three architectural layers (business, IS, IT), and (3) analyzing the strategy space without considering any future constraints of operations (strategy-operations). This means that the CHOOSE metamodel needs to define concepts for each of the four essential focuses, that the metamodel concepts may represent elements related to business, IS and IT, and that CHOOSE models can be constructed for representing and analyzing enterprise strategy without being constrained by the current operations, so that strategy (needs) and operations (means) are not mixed.

\section{Initial Metamodel}

We will first explain why the metamodel of the KAOS approach was chosen as a starting point for designing the CHOOSE metamodel. Next, the KAOS metamodel itself will briefly be presented. A more detailed description is provided in Appendix 1.

\subsection{KAOS as a Starting Point}

In addition to the EA approaches listed in Table 1, we also investigated goal-oriented requirements engineering (GORE) approaches. The main reason for choosing KAOS as a starting point is that from the investigated EA and GORE approaches, only KAOS (Van Lamsweerde 2009) and EKD (Stirna and Persson 2007) are explicitly built around the four essential EA focuses. Furthermore, KAOS was preferred to EKD as its metamodel is formally defined, which helps provide precise definitions for the concepts in the CHOOSE metamodel. The KAOS metamodel also explicitly distinguishes between concepts related to strategy and concepts related to operations. On the other hand, it should be pointed out that KAOS is not an original EA approach, but rather a requirements engineering approach intended to model systems. Therefore, its selection as the initial metamodel for CHOOSE was not trivial and had to be based on well-reasoned considerations, as explained below.

Engelsman et al. (2011) wrote an interesting paper on the use of GORE in EA in order to deal with the problem that current EA frameworks offer little support for modelling the underlying motivation of EAs in terms of stakeholder concerns and the high-level goals addressing these concerns. Their work lay at the basis of the ArchiMate 2.0 standard for EA modelling that extended ArchiMate 1.0 with a motivational extension (The Open Group 2012). The need for (a 
simple version of) goal refinement in EA approaches was confirmed after tests in case studies (Engelsman and Wieringa 2012). Therefore, GORE approaches were also considered as candidates for the selection of the initial metamodel, apart from the EA approaches listed in Table 1.

Well-known GORE techniques are $i^{*}(\mathrm{Yu}$ 1993) and KAOS (Dardenne et al. 1991; van Lamsweerde et al. 1991). KAOS is a requirements engineering approach for software-intensive systems within an organizational or physical environment (Van Lamsweerde 2009). It is important to stress that KAOS is primarily intended to model organizational or physical systems based on goals and requirements, rather than used to model software. However, since enterprises are regarded as systems within EA (Jonkers et al. 2006), they can also be modelled as systems with KAOS. Compared to $i^{*}$, which is more focused on the early requirements engineering phase and the modelling of dependencies between actors (Engelsman et al. 2011), KAOS has an important advantage since it makes it possible to make a broader overview of a system within its environment.

The ultimate choice for KAOS was, however, based on its great fit with the essential EA dimensions that we identified after analyzing important EA frameworks (see section 4). First of all, its metamodel is based on four viewpoints that provide a one-to-one mapping with the four essential EA focuses. Second, KAOS models systems that can be composed of business (or realworld), software, data and technology components, so a blend of the three architectural layers can be used. Third, since KAOS is a GORE approach, it provides a means to analyze the strategy space without anticipating any constraints of operations. In GORE, abstract higher-level goals are gradually refined to more concrete lower-level goals, which are used to specify requirements for systems (Anton 1996; Anton et al. 1994; Dardenne et al. 1993). These goals, which are part of the why-focus, are then linked to operations, which are part of the how-focus, in order to maintain traceability (Mostow 1985). Engelsman et al. (2011) state that a company is a good example of a system and goals can be a good basis for modelling the motivational dimension of a company. Other research concludes that business goals form an integral part of enterprise models (Boman et al. 1997; Loucopoulos and Kavakli 1995).

A final motivation for choosing KAOS is that its metamodel is well elaborated after more than twenty years of research, and is hence a good starting point to reuse existing knowledge.

\subsection{KAOS Metamodel}

The KAOS metamodel consists of four main viewpoints that define different sub-models (Fig. 5): goal, agent, operation, and object. These viewpoints are mapped onto the four essential EA focuses of why, who, how and what:

- Goal viewpoint (why-focus), where goals are refined and justified until a goal hierarchy has been put together for tackling a particular problem.

- Agent viewpoint (who-focus), in which agents are assigned to the goals they are responsible for.

- Operation viewpoint (how-focus), which defines various behaviours that the agents need to fulfil their requirements. 
- Object viewpoint (what-focus), which is used to define and document the objects (i.e. entities, agents, and associations).

There is an additional viewpoint (not shown in Fig. 5), which completes the static representation of system functionalities by capturing the required system dynamics. This behaviour viewpoint defines sub-models that can be represented using UML sequence diagrams and state diagrams. The concepts used in these sub-models are most closely related to the when Zachman focus, but this is not among the essential focuses of EA frameworks that we identified. Hence, it is clear that the behaviour viewpoint is not essential for EA modelling and can therefore be left out of the initial metamodel.

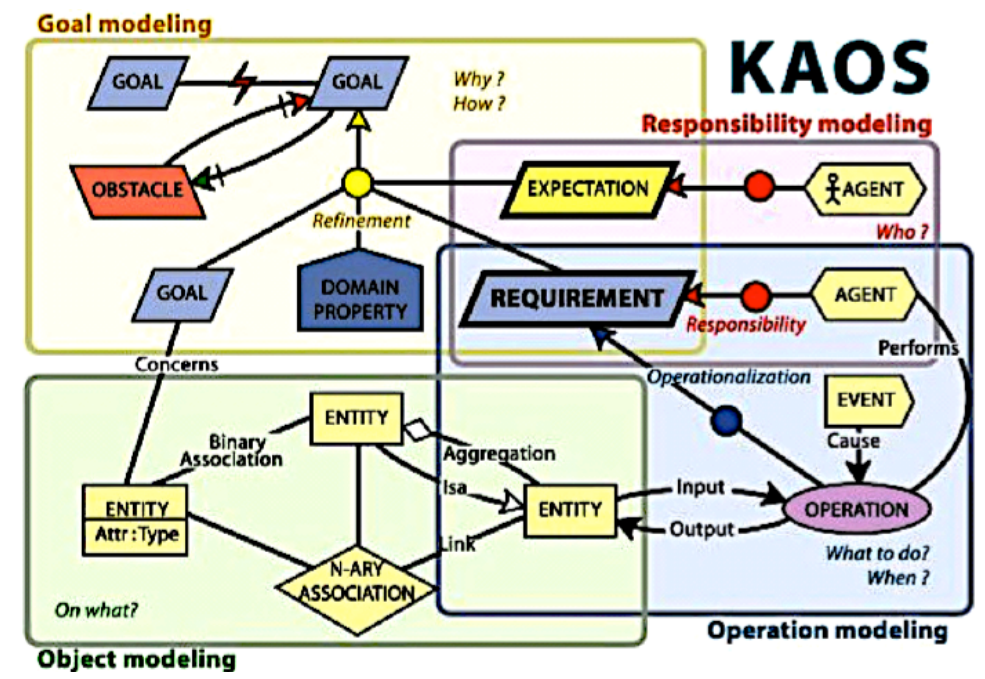

Fig. 5 A simplified overview of the KAOS metamodel (from (Respect-IT 2007))

In the remainder of the paper, concepts from the goal, agent, operation, and object viewpoints will further be coloured in yellow, red, purple, and green, respectively. Definitions can be found in Appendix 1 and will be provided in the remainder of the paper when relevant to the discussion regarding the changes made during the action research and case studies (see next section).

\section{From KAOS to CHOOSE}

The most important change to transform the KAOS metamodel into the CHOOSE metamodel entailed deleting the elements that were not further used after the feasibility test of the full KAOS metamodel in the SME (Bernaert and Poels 2011) and were not asked for in the following rounds. As Moody (2003) mentioned, adoption is related to both effectiveness (i.e. benefits) and efficiency (i.e. costs). In order to develop CHOOSE, we first focused on efficiency and started with the essential part of an EA approach. During the action research in the SME, we then found out which parts had to be added for which the increase in effectiveness (i.e. increase in benefits) was larger than the decrease in efficiency (i.e. increase in costs).

Firstly, it is important to note that only two meta-attributes are mandatory for any meta-concept of all viewpoints in KAOS: Name and Def. These meta-attributes are also the attributes of the four central CHOOSE concepts; all other KAOS meta-attributes were omitted. Def was changed into a less formal Description attribute. This attribute has to be comprehensive and precise, yet also needs to provide a clear, though informally stated, description in natural language. 
Secondly, other parts were omitted, changed, or added in each viewpoint and will be discussed for each viewpoint in the next sections. As mentioned before, after the action research was set up in the SME, five more case studies in SMEs with different characteristics were performed. Therefore, sometimes a particular change was triggered by a problem experienced with the use of CHOOSE in a case study company. If a similar problem was noticed in the action research SME, the solution chosen for the case study company was also evaluated in the action research SME, and after a positive evaluation this solution was then also incorporated into the metamodel.

In Appendix 1, a visual overview is given of the transformation of the KAOS metamodel into the CHOOSE metamodel (Fig. 11 to Fig. 13). It is important to note that the following discussion is based on the complete KAOS metamodel (Fig. 11 in Appendix 1) and not on the simplified overview presented in the previous section (Fig. 5). In the following sections, changes to the different viewpoints will be discussed.

\subsection{Goal Viewpoint}

The Goal concept was retained together with the concept of Refinement. The attribute of Refinement became $I d$, in order to enable the SME to distinguish between alternative Refinements. The distinction between BehaviouralGoals and SoftGoals was omitted, because the SME was not interested in qualitative (Mylopoulos et al. 1992) nor quantitative (Letier and van Lamsweerde 2004) analyses, because of time constraints.

The same holds for the Obstacle analysis. This part was left out because it was never used as such. In the SME, Obstacles were implicitly addressed by naming the Goals according to the problem they aim to resolve (e.g., "decrease out of stock situations") instead of using Obstacles (e.g., "out of stock situation") and then Resolving them by means of a Goal. However, the SME wanted to model conflicting Goals of different stakeholders of the company in order to resolve these conflicts. The Conflict relationship between Goals was thus retained and explicitly represented by a relationship in the CHOOSE metamodel.

DomDescript was never used as it corresponds in KAOS to physical laws that cannot be broken. This aspect relates to formulating business rules in the context of an enterprise as part of an EA model. For example, an SME can express that a specific bank account (Object) can only be Controlled by maximum three Human Actors. As no business rules have been expressed so far in the SME, this concept of business rules is not yet explicitly represented in the CHOOSE metamodel. Nevertheless, the tool support we are developing for CHOOSE (Bernaert et al. 2013a; Dumeez et al. 2013; Ingelbeen et al. 2013) does make it possible to check such rules, by means of queries of the model level.

Finally, there is no longer an explicit distinction between Expectations and Requirements in CHOOSE. Since Actors can be different types, these types of Goals can simply be queried from the CHOOSE model to determine which Goals are from a specific type of Actor.

\subsection{Agent Viewpoint}

The Agent concept was renamed into Actor, so that it would be more consistent with the terminology used in most EA frameworks (Table 1). The distinction between SoftwareToBeAgent and EnvironmentAgent was turned into a distinction between Human Actor, Role, Software Actor, 
and Device (hardware and equipment), which were only implicitly present in the KAOS metamodel via the optional Category attribute of an Agent. This change was not initiated by the SME, but rather by the need to adapt KAOS so that it would support the EA essential dimensions better (see section 4.2) and be able to model a blend of the three architectural layers (business, IS, IT). The SME used the distinction between these types of Actors sometimes but not all the time, in order to speed up the modelling task. As a consequence, the specialization became optional in CHOOSE.

The SME experienced problems linking Operations to functions, for which a solution had to be found. Sometimes, functions appeared to switch between Human Actors, depending on the availability of the actors themselves, as well as their available time. The use of Roles and Human Actors that Perform Roles is briefly mentioned in KAOS, but not explicitly present in its metamodel. Still, as this is widely supported by EA frameworks (see Table 1), it was explicitly added to the CHOOSE metamodel so that this issue could be addressed.

The reflexive Supervision relationship was added between Human Actors because the SME immediately became aware of the need to make organizational charts. A many-to-many Supervision relationship was chosen to also enable matrix organizational structures in which a supervisee can have more than one supervisor.

Another reflexive relationship between Actors, Aggregation, was initially not included in the metamodel. However, one of the SMEs in which we performed case study research (to design the CHOOSE method, see section 3) had 37 employees and the metamodel did not allow us to group Actors into departments or other categories, because such units are neither Human Actors nor Roles. In order to be able to group Actors according to different levels of granularity (e.g., business unit or department), which is also common in EA frameworks (Table 1), the reflexive Aggregation relationship was thus added again. However, the SME did not express the need to make a further specialization of Actor in department or business unit. Therefore, the specialization of Actor in its subtypes is not covering (incomplete), since an Actor can be something other than a Human Actor, a Role, a Software Actor, or a Device. The problem of not being able to group Actors was initially not brought to the attention in the action research SME, because this is an SME with only six employees. When the SME discovered in the second additional action research cycle that it could model its organizational chart more precisely, it fully supported this change in the metamodel.

At first, only one type of relationship was retained between Goals and Actors (i.e. Assignment). However, this soon became insufficient, because the relationship was used to assign Actors to Goals (as executing Actor) for lower-level Goals, but was also incorrectly used to express that an Actor "wanted" a Goal to be fulfilled for higher-level Goals. Therefore, the Wish relationship between Actors and Goals, only implicitly present in the KAOS metamodel as an attribute of Agent, was made explicit in the CHOOSE metamodel as a relationship. This was usually on a higher Goal level than the Assignment relationship between both.

The Assignment relationship, however, has a different meaning than the relationship in KAOS. In KAOS, an Assignment relationship makes it possible to OR-Assign different Agents to the same Goal, while only one Agent can be made Responsible of that Goal. In CHOOSE, Actors have an Assignment relationship with a Goal if they have been instructed to achieve that Goal (i.e. they are 
IOR-assigned). This enabled the SME to assign multiple Actors to the same Goal, which was more in line with the SME's business reality. Whether or not the Actors were responsible for that Goal at a specific time, there were more ad-hoc decisions and there was no need for this to be expressed in the metamodel. The SME also Assigned some Actors to non-LeafGoals so that the model would reflect reality more clearly. Yet, this is not possible in the original KAOS metamodel. This is a subtle, yet important difference between KAOS and CHOOSE: in KAOS, Goals have to be Refined until they can be under the Responsibility of just one Agent. These LeafGoals can then be Operationalized by one or more Operations. The Operations also have to enable Performance by just one Agent. In contrast, in CHOOSE, Goals at any level can be assigned to Actors and can be Operationalized by Operations that can be Performed by more than one Actor. This clearly reflects the real-life organizational levels that can exist in a company. Nevertheless, some consistency problems still occurred in the SME due to this adaptation, for which additional OCL constraints (Appendix 2 Table 3: constraints 5-6, 21-22) were added.

At the start, only one relationship was retained between Actor and Object to express that an Object belonged to an Actor. However, this relationship could be more correctly modelled with an Association between an Actor and Entity in the object viewpoint if Actor was seen as a subtype of Object. The relationship between Actor and Object was therefore omitted and Actor was kept as a subtype of Object and could be used in the object viewpoint by the SME. Thus, the CHOOSE metamodel did not contain any extra relationship between Actor and Object anymore. One of the case study SMEs did a lot of administrative work and some discussions arose based on read and write rights of documents. This problem did not occur in the action research SME at first. When the EA model of the action research SME became more complete, it did become an issue as the action research SME also wanted to express the confidentiality of financial data. For example, it had to be decided who could see a particular bank account and who could make payments. In order to solve this problem, some options were considered and most often, a distinction between creating (if the object is newly created), transforming (if it is changed), and using (if it is only used and not modified) was found to be of importance. The SME did not make a distinction between creating and transforming. Therefore, the Monitoring and Control relationships from KAOS were added again, but instead of linking them to Associations and Attributes, we provided a direct link from Actors to Objects (Fig. 11). This was a logical step, because the SME did not specify any additional Attributes for Objects and because Associations are still subtypes of Objects. As a result, if an Association has to be explicitly Monitored or Controlled, the Association can be objectified, and Actors in CHOOSE can thus Monitor and/or Control an Object.

Finally, Dependencies between Actors can be queried from the CHOOSE model, and were therefore omitted from the metamodel. The assumption behind this is that if Actors are Assigned to the same Goal, or if they have to Perform the same Operation, they are dependent on each other.

\subsection{Operation Viewpoint}

The operation viewpoint differs significantly between KAOS and CHOOSE. As mentioned before, an Operation in KAOS can only have a Performance relationship with exactly one Agent. However, when more Operations were added to the SME's EA model during the action research, there was no clear overview anymore. A solution to this problem was found by examining how 
ARIS (Scheer 2000) and BPMN (OMG 2011a) structure processes. A reflexive Includes relationship was added to enable the SME to make Operations part of other(s) in order to make it possible to create a structured Operation overview (sometimes called a map or landscape). Some constraints (Appendix 2 Table 3: constraints 9, 11, 22, 25) were adapted or added to maintain consistency.

It has already been pointed out that process modelling should not be included in EA. The SME, for its part, did not feel the need to make any process descriptions either. Still, some SMEs are likely to be confronted with this need for process modelling if standardization becomes more important (Ross et al. 2006). To make sure that they have some kind of EA overview of Processes at their disposal, a Process overview is included, while detailed process modelling is left out of the CHOOSE metamodel. However, process modelling descriptions can easily be linked to this Process overview of CHOOSE (e.g., with attachments in the software tool), which has as an advantage that the choice of process modelling language (e.g., BPMN, EPC, UML activity) can be made based on the SME's preferences, without this affecting the CHOOSE metamodel.

The name Operation was retained, since there is a clear distinction in a business context between a Process (Weske 2012) and a Project (Kerzner 2013), which was confirmed by the action research SME. A Process will typically be performed multiple times, while a Project is performed only once and has time, budget, and other constraints. In CHOOSE, an Operation can therefore either be a Process or a Project. The SME had some Projects that could be quite disruptive for their business and wanted to treat these Projects differently than the Processes (e.g., some milestones were formulated for these Projects). Therefore, the SME sometimes, but not always, wanted to distinguish between a Process and Project. That is why the specialization is optional: if not further specified, the SME is not interested in making the difference between a Process and Project.

The Performance relationship helped the SME to make a load analysis of all Operations linked to an Actor. However, this load analysis needed some corrections, because for example sometimes the Actor would only be informed about the Operation once in a while, which was less timeconsuming than being held responsible for it. For this problem, different solutions exist (e.g., RACI, RASCI, CAIRO). In order to be able to use a RACI (Responsible, Accountable, Consulted, and Informed) labelling of the Performance relationships, an association class including the attribute Type was added to the Performance relationship. This RACI chart is also used, for example, by the IT governance reference framework COBIT to define responsibilities (ISACA 2012). Working with a generic Type attribute instead of a specific set of labels makes the modelling effort much more flexible, so that the SME may choose another responsibility assignment matrix. The load analysis during the action research would then be more accurate, based on the different Types of Performance relationships between Actors and Operations.

As the SME linked Operations with Goals - not only LeafGoals - at different levels, the Operationalization link needed to be adapted. A constraint (Appendix 2 Table 3: constraint 9) was added to maintain consistency. However, it is best to delay the Operationalization of a Goal as long as possible, to make sure that the constraints of operations are still disregarded during the analysis of the strategy space, which is an essential element in EA frameworks (Table 1). This 
aspect could be further investigated with regard to the future development of the CHOOSE method, but is beyond the scope of the present paper.

The Input and Output links between Operations and Objects were retained for the same reasons as the Monitor and Control links between Actors and Objects: to give the SME the possibility to express which Objects are the Input (i.e. using) of an Operation and which ones are Output (i.e. creating or transforming). These relationships were also directly linked to Objects instead of Associations and Attributes (Fig. 11). An Object that is the Input of an Operation was often a resource in the SME, while an Object that is Output was often a product of the SME. However, as there are multiple exceptions, this was not included in the metamodel. For example, for more administrative Operations documents were sometimes needed as Input, in which case the Output would be an invoice, for example.

\subsection{Object Viewpoint}

The object viewpoint was less used than the other three viewpoints. The SME only needed to model Objects and the Associations between them. Only if more specificity was required, was an Object further broken down into Entity, Actor or Association. As a consequence, this specialization could be optional. There was no need to include extra Attributes, DomDescripts or DomInits either, because the Description attribute of an Object was sufficiently specific and the CHOOSE metamodel is not focused on precise system specification like KAOS. The Event concept, referred to in this object viewpoint but part of the behavioural viewpoint in KAOS, was also omitted. This can again be accommodated by process modelling languages and state diagrams.

In the SME of the action research, only Associations that Link two Objects were used. To enhance semantic clarity, Associations between more than two Objects were disregarded. The SME did not use any specific Attributes for Objects, nor did it define ApplicationSpecific Associations. Instead, the two attributes of an Association - Name and Description because an Association is a subtype of an Object - were sufficient to clearly describe the different Associations.

Aggregation and Specialization were first hardly used. However, when the CEO of the SME tried to specify a bill of materials (for example, by asking himself which car parts could be replaced by the SME), the Aggregation relationship offered a good solution (Hegge and Wortmann 1991). The same happened when the CEO tried to get a product overview (for example, by asking himself how the SME sorts the warehouse according to tyre type), the Specialization relationship was a good solution (Eriksson and Penker 2000). A good method to explain these options can also be recommended. Preferably, this explanation does not use the terms Aggregation and Specialization, which were unknown to the CEO in this particular case. The choice to specify an Association as either an Aggregation or Specialization is an Optional, disjoint (Or) choice (OMG 2011c). This means that an Association does not have to be further specified in CHOOSE if the SME does not need it, but if it is, it can only be one of the subtypes.

The Concern relationship between Goal and Object was retained, although the SME in fact did not use it frequently. Further research in more SMEs could give more insight into the use of this relationship, for example in order to detect consistency conflicts (Appendix 2 Table 3: constraint 12). 
An Object can be Input and Output of the same Operation, as its State can be changed by an Operation (for example, the customer file that was updated in the SME). However, there was no need to explicitly model these States. This is in line with the choice to also exclude process modelling from the CHOOSE metamodel, because this can also be achieved by process modelling languages and state diagrams.

\section{CHOOSE Metamodel}

\subsection{Complete CHOOSE Metamodel}

The CHOOSE metamodel was robust after the third action research cycle and no further changes needed to be made during the fourth cycle. According to the Object Management Group (OMG) (2013, 2012b) standards, the metamodel presented in this research is a computation independent model (CIM) at M2-level. Since it is described as a unified modelling language (UML) class diagram (OMG 2011b, c), this model can also serve as a platform independent model (PIM) for software tool support development (Bernaert et al. 2013a; Dumeez et al. 2013; Ingelbeen et al. 2013). The models made with this metamodel, and thus instantiating it, will be at M1-level and will be EA models for the specific SME being modelled.

Fig. 6 shows this final CHOOSE metamodel, including all optional parts. Actor is represented twice for clarity's sake, but refers to the same concept.

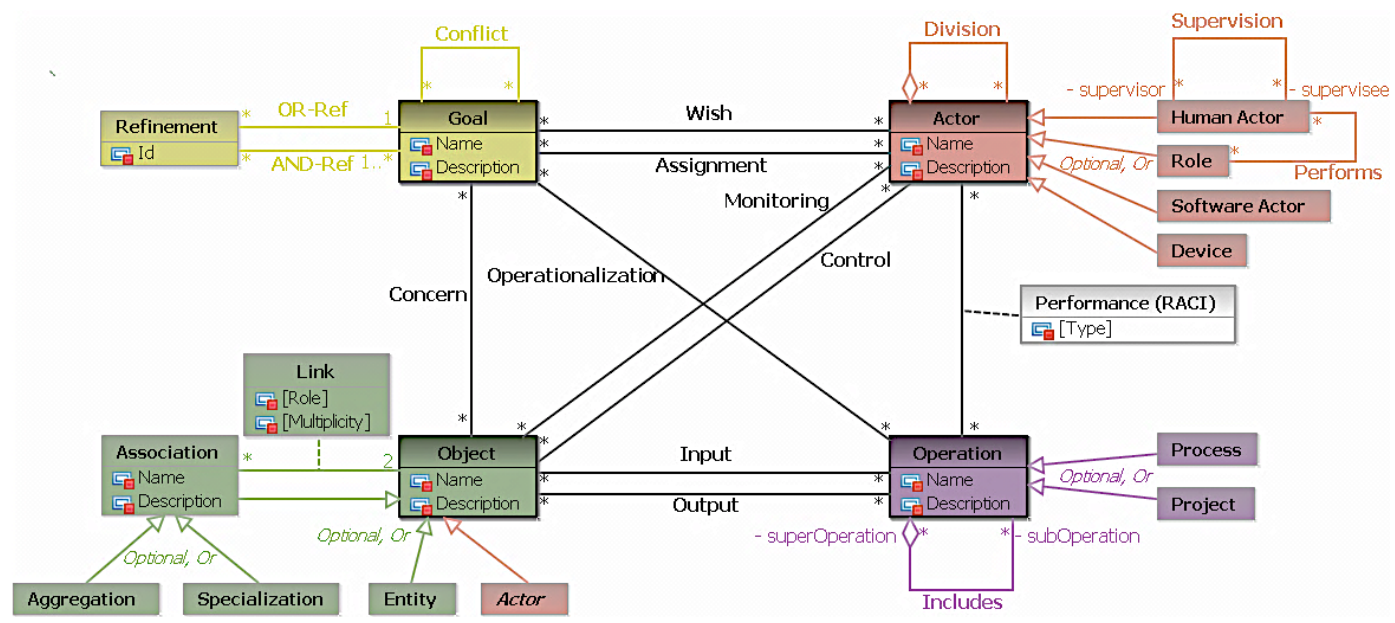

Fig. 6 CHOOSE metamodel

\subsubsection{CHOOSE Goal Viewpoint}

Goal is the central concept in the goal viewpoint and has the attributes Name and Description. A Goal can have a Conflict relationship with zero or more other Goals.

An OR-Ref links one higher-level Goal with a Refinement. If different Refinements are linked via OR-Ref links to the same higher-level Goal, this means the Goal is OR-Refined several times. If only one Refinement is linked via an OR-Reflink to a higher-level Goal, this means the Goal can only be refined in one possible way. Each Refinement is then linked via $A N D$-Ref links with one or more lower-level Goals. This implies each alternative Refinement of a higher-level Goal is linked via $A N D$-Ref links with one or more lower-level Goals, which all have to be fulfilled in order to meet the higher-level Goal with which this Refinement is linked via an OR-Ref link. A special case 
is when a higher-level Goal is OR-Refined by just one Refinement and this Refinement is ANDRefined by just one lower-level Goal. In this case, the higher-level Goal is simply refined by the lower-level Goal. If a Refinement thus only has one upper Goal (OR-Ref) and one lower Goal $(A N D-R e f)$, it can be seen as a single refinement of a higher-level Goal in a lower-level Goal. A Goal does not have to have a link with a higher Refinement (reached through an AND-Reflink) if it is one of the highest-level Goals in the Goal hierarchy, and it does not have to have a lower Refinement (reached through an OR-Ref link) if it is one of the lowest-level Goals in the hierarchy (i.e. a leaf Goal).

A Goal can have a Wish or Assignment relationship with zero or more Actors, can be Operationalized by zero or more Operations, and can have a Concern relationship with zero or more Objects.

\subsubsection{CHOOSE Actor Viewpoint}

Actor is the central concept in the actor viewpoint and has the attributes Name and Description. An Actor can be an aggregation of zero or more other Actors and can be part of zero or more Actors via a Division relationship. However, if an Actor is a Human Actor, it cannot be an aggregation of other Actors (Appendix 2 Table 3: constraint 14) and other relevant constraints to limit the Aggregation of different Actor types are added (Appendix 2 Table 3: constraints 15-19). An Actor can, but does not have to be (i.e. it is Optional) Specialized in either (disjoint Or) a Human Actor, a Role, a Software Actor, or a Device. A Human Actor can be Supervised by zero or more supervisors, or can Supervise zero or more supervisees. A Human Actor can Perform zero or more Roles, while a Role can be Performed by zero or more Human Actors.

Actors can have a Wish (only unspecialized Actors or Human Actors, see Appendix 2 Table 3: constraint 4) or Assignment relationship with zero or more Goals, they can have a Performance relationship (some kind of RACI or other Type) with zero or more Operations, and they can Monitor or Control zero or more Objects.

\subsubsection{CHOOSE Operation Viewpoint}

Operation is the central concept in the operation viewpoint and has the attributes Name and Description. An Operation can be Included in zero or more other superOperations and can Include zero or more subOperations. An Operation can, but does not have to be (i.e. it is Optional) Specialized in either (disjoint $O r$ ) a Process or a Project.

An Operation can Operationalize zero or more Goals, can have a Performance relationship (some kind of RACI or other Type) with zero or more Actors, and can have zero or more Objects as Input or Output.

\subsubsection{CHOOSE Object Viewpoint}

Object is the central concept in the object viewpoint and has the attributes Name and Description. An Object can, but does not have to be (i.e. it is Optional) Specialized in either (disjoint $O r$ ) an Entity, Actor, or Association. An Association Links two Objects, while an Object can have zero or more Associations with one other Object. An Association inherits the attributes Name and Description, which are also visualized for clarity's sake, and a Link has the optional attributes Role 
and Multiplicity. An Association can, but does not have to be (i.e. it is Optional) Specialized in either (disjoint Or) an Aggregation or a Specialization.

An Object can have a Concern relationship with zero or more Goals, can be Monitored or Controlled by zero or more Actors, and can be Input or Output for zero or more Operations.

\subsection{Core Part of the CHOOSE Metamodel}

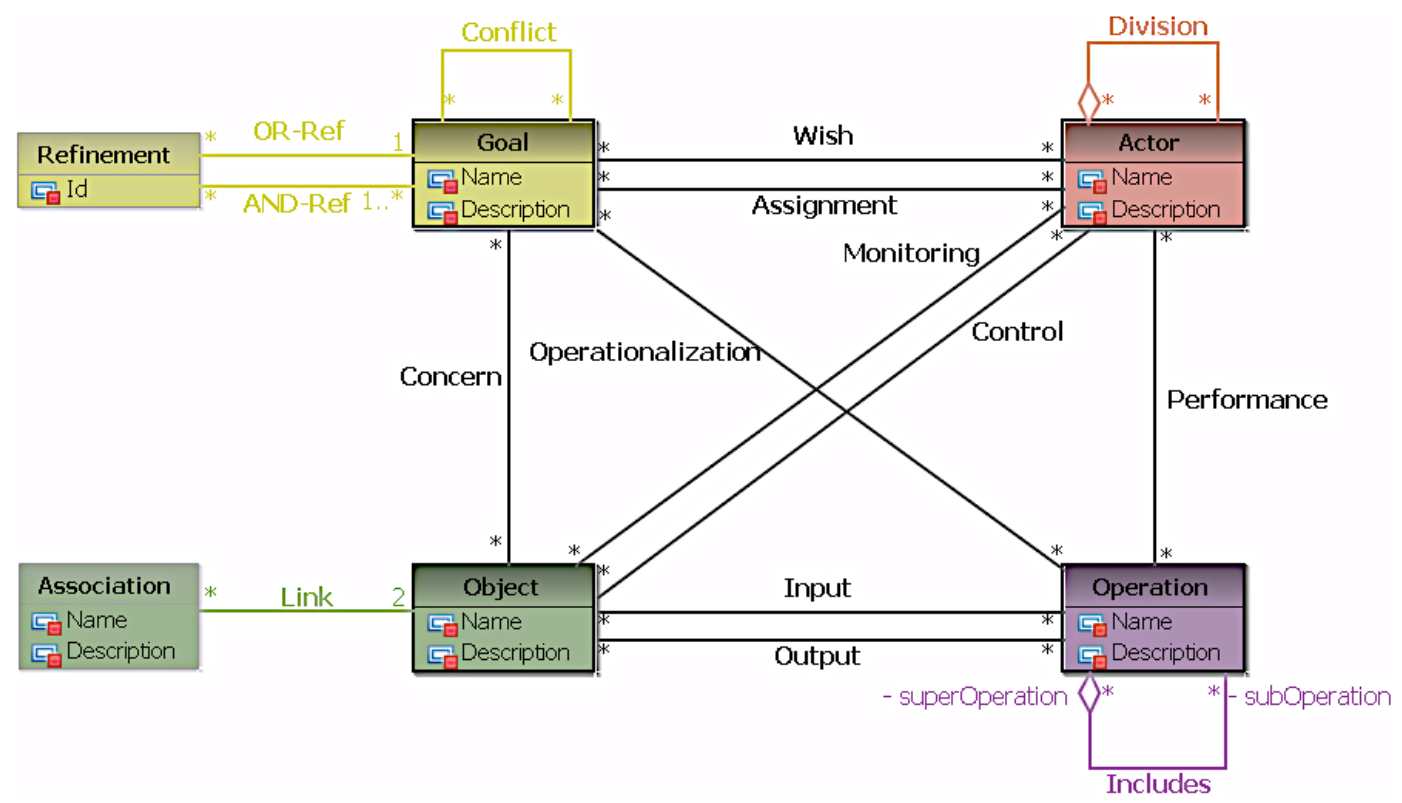

Fig. 7 Core part of the CHOOSE metamodel

Fig. 7 shows the core part of the CHOOSE metamodel, which only includes the minimum set of concepts and relationships of the CHOOSE metamodel required to model an SME's EA. The optional specializations and attributes are left out of this core representation of the CHOOSE metamodel, since they were only useful in some scenarios for the action research SME and do not belong to the essential EA dimensions.

These minimal parts of the CHOOSE metamodel can be used by SMEs to quickly and easily create an EA model. If needed, however, an Actor could then for example later on be specialized as a Human Actor in order to use the Supervision relationship of the complete CHOOSE metamodel. For instance, if the SME treats a Process differently than a Project, it could use the extensions of the complete metamodel and specialize the Operations into Processes and Projects. This core part of the CHOOSE metamodel represents the bare minimum, while still conforming to the EA essentials from section 4.2.

\subsection{CHOOSE Definitions Using SBVR}

In order to decrease misunderstandings, formal definitions are provided to contribute to the unambiguous definition of the CHOOSE concepts. Not all concept definitions in the CHOOSE metamodel could be retained from KAOS, since KAOS is used for system specification, while CHOOSE is used to make EA models of SMEs. Hence, the context in which the concepts are used is different.

When the KAOS definitions had to be adapted, the first choice was to relate the definitions, if possible, to ArchiMate definitions for two reasons. First, ArchiMate has been adopted by The 
Open Group (2012) as a standard and second, in future research, CHOOSE will be mapped onto ArchiMate to make bidirectional translation possible (Roose et al. 2013). For the organizational chart, definitions are linked to OMG's (2009) organization structure metamodel (OSM), since this metamodel is a widely used standard. The Project definition is adapted from the project management body of knowledge (Project Management Institute 2013) and the Process definition from OMG's (2011a) BPMN and ArchiMate (The Open Group 2012). Finally, the concepts derived from KAOS that cannot be related to a relevant EA definition are taken from the original KAOS definition (Van Lamsweerde 2009).

In Table 2, the definitions of the entities and relationships of CHOOSE are explained by means of SBVR (OMG 2008). Only the business vocabulary part of SBVR is used, as the rules are expressed in OCL (section 7.4).

Table 2 CHOOSE entities and relationships defined with SBVR

\begin{tabular}{|c|c|c|}
\hline CONCEPT & DEFINITION & SOURCE \\
\hline \multicolumn{3}{|l|}{ Object Type } \\
\hline Goal & $\begin{array}{c}\text { An end state that an actor wishes to achieve and that is to } \\
\text { be brought about or sustained through appropriate } \\
\text { operations. }\end{array}$ & $\begin{array}{l}\text { Goal (The Open Group } \\
\text { 2012; OMG 2010) }\end{array}$ \\
\hline Refinement & $\begin{array}{l}\text { Groups lower-level goals that all have to be fulfilled in } \\
\text { order to fulfil a higher-level goal. Different refinements } \\
\text { for one higher-level goal express different alternatives. }\end{array}$ & $\begin{array}{l}\text { Refinement (Van } \\
\text { Lamsweerde 2009) }\end{array}$ \\
\hline Actor & $\begin{array}{c}\text { An organizational entity that is capable of performing } \\
\text { operations. }\end{array}$ & $\begin{array}{l}\text { Business actor (The Open } \\
\text { Group 2012) }\end{array}$ \\
\hline Human Actor & A human being who is capable of performing operations. & $\begin{array}{l}\text { Human actor (The Open } \\
\text { Group 2012) }\end{array}$ \\
\hline Role & $\begin{array}{l}\text { The responsibility for the performance of specific } \\
\text { operations, to which a human actor can be assigned who } \\
\text { performs the role. }\end{array}$ & $\begin{array}{l}\text { Business role (The Open } \\
\text { Group 2012) }\end{array}$ \\
\hline $\begin{array}{l}\text { Software } \\
\text { Actor }\end{array}$ & $\begin{array}{l}\text { A software system or part of a software system that } \\
\text { encapsulates its behaviour and data to perform } \\
\text { operations. }\end{array}$ & $\begin{array}{l}\text { Business actor }+ \text { Application } \\
\text { component (The Open } \\
\text { Group 2012) }\end{array}$ \\
\hline Device & $\begin{array}{l}\text { A hardware resource or physical equipment that is } \\
\text { capable of performing operations. }\end{array}$ & $\begin{array}{l}\text { Business actor }+ \text { Device } \\
\text { (The Open Group 2012) }\end{array}$ \\
\hline Operation & $\begin{array}{l}\text { Internal behaviour that needs objects as input and } \\
\text { produces objects as output, in order to operationalize } \\
\text { goals. It can be a process or project. }\end{array}$ & $\begin{array}{l}\text { Adapted from Operation } \\
\text { (Van Lamsweerde 2009) }\end{array}$ \\
\hline Process & $\begin{array}{l}\text { A behaviour element that groups behaviour based on an } \\
\text { ordering of activities with the objective of carrying out } \\
\text { work. It is intended to produce a defined set of products } \\
\text { or business services. }\end{array}$ & $\begin{array}{c}\text { Process (OMG 2011a) }+ \\
\text { Business process (The Open } \\
\text { Group 2012) }\end{array}$ \\
\hline Project & $\begin{array}{l}\text { A temporary endeavour undertaken to create a unique } \\
\text { product, service or result. }\end{array}$ & $\begin{array}{c}\text { Project (Project } \\
\text { Management Institute 2013) } \\
\end{array}$ \\
\hline Object & $\begin{array}{l}\text { A passive element that has relevance from a business, } \\
\text { information, or technological perspective. It corresponds } \\
\text { to a real world counterpart that may or may not be } \\
\text { physical. }\end{array}$ & $\begin{array}{l}\text { Business object (The Open } \\
\text { Group 2012) + Object } \\
\text { (Snoeck et al. 1999) }\end{array}$ \\
\hline Entity & An autonomous and passive object. & $\begin{array}{l}\text { Entity (Van Lamsweerde } \\
\text { 2009) }\end{array}$ \\
\hline \multicolumn{3}{|l|}{ Fact Type } \\
\hline OR-Ref & Refines a higher-level goal in alternative refinements. & $\begin{array}{l}\text { OR-refinement (Van } \\
\text { Lamsweerde 2009) }\end{array}$ \\
\hline AND-Ref & $\begin{array}{l}\text { Expresses that an alternative refinement of a higher-level } \\
\text { goal can be satisfied by satisfying all its subgoals. }\end{array}$ & $\begin{array}{l}\text { AND-refinement (Van } \\
\text { Lamsweerde 2009) }\end{array}$ \\
\hline Conflict & $\begin{array}{c}\text { Interconnects goals to capture potential conflicts among } \\
\text { them. }\end{array}$ & $\begin{array}{l}\text { Conflict (Van Lamsweerde } \\
\text { 2009) } \\
\end{array}$ \\
\hline Wish & $\begin{array}{l}\text { Captures the fact that an actor would like a goal to be } \\
\text { achieved. }\end{array}$ & $\begin{array}{l}\text { Wish (Van Lamsweerde } \\
\text { 2009) }\end{array}$ \\
\hline
\end{tabular}




\begin{tabular}{|c|c|c|}
\hline Assignment & $\begin{array}{l}\text { An actor is assigned to a goal if it is required to restrict } \\
\text { its behaviour so as to achieve the goal. }\end{array}$ & $\begin{array}{l}\text { Responsible (Van } \\
\text { Lamsweerde 2009) }\end{array}$ \\
\hline $\begin{array}{l}\text { Operationali- } \\
\text { zation }\end{array}$ & $\begin{array}{l}\text { Refers to the process of mapping goals (ends) to } \\
\text { operations (means) realizing them. }\end{array}$ & $\begin{array}{l}\text { Operationalization (Van } \\
\text { Lamsweerde 2009) }+ \\
\text { Realization (The Open } \\
\text { Group 2012) }\end{array}$ \\
\hline Concern & Connects goals to the objects to which they refer. & $\begin{array}{c}\text { Concern (Van Lamsweerde } \\
\text { 2009) } \\
\end{array}$ \\
\hline Division & Indicates that an Actor groups a number of other Actors. & $\begin{array}{l}\text { Aggregation (The Open } \\
\text { Group 2012) }\end{array}$ \\
\hline Supervision & A supervisee reports to a supervisor. & Supervises (OMG 2009) \\
\hline Performs & Links roles with human actors that fulfil them. & $\begin{array}{l}\text { Assignment (The Open } \\
\text { Group 2012) }\end{array}$ \\
\hline $\begin{array}{l}\text { Performance } \\
\quad(\mathrm{RACI})\end{array}$ & $\begin{array}{l}\text { Links operations with active elements (actors) that } \\
\text { perform them or more specifically that are responsible, } \\
\text { accountable, consulted, or informed. }\end{array}$ & $\begin{array}{l}\text { Assignment (The Open } \\
\text { Group 2012) + RACI } \\
\text { (ISACA 2012) }\end{array}$ \\
\hline Monitoring & $\begin{array}{l}\text { An actor monitors an object if it can use the object, } \\
\text { without changing it. }\end{array}$ & $\begin{array}{c}\text { Monitoring (Van } \\
\text { Lamsweerde 2009) }\end{array}$ \\
\hline Control & An actor controls an object if it can create or transform & $\begin{array}{l}\text { Control (Van Lamsweerde } \\
\text { 2009) }\end{array}$ \\
\hline Includes & $\begin{array}{l}\text { Groups suboperations in the superoperations of which } \\
\text { they are part. }\end{array}$ & $\begin{array}{l}\text { Aggregation (The Open } \\
\text { Group 2012) }\end{array}$ \\
\hline Input & Designates an object to which the operation applies. & $\begin{array}{l}\text { Input (Van Lamsweerde } \\
\text { 2009) }\end{array}$ \\
\hline Output & Designates an object on which the operation acts. & $\begin{array}{l}\text { Output (Van Lamsweerde } \\
\text { 2009) }\end{array}$ \\
\hline Association & $\begin{array}{l}\text { Models a relationship between objects that is not } \\
\text { covered by another, more specific relationship. }\end{array}$ & $\begin{array}{l}\text { Association (The Open } \\
\text { Group 2012) } \\
\end{array}$ \\
\hline Aggregation & $\begin{array}{c}\text { Indicates that an object groups a number of other } \\
\text { objects. }\end{array}$ & $\begin{array}{l}\text { Aggregation (The Open } \\
\text { Group 2012) } \\
\end{array}$ \\
\hline Specialization & $\begin{array}{c}\text { Indicates that an object is a specialization of another } \\
\text { object. }\end{array}$ & $\begin{array}{l}\text { Specialization (The Open } \\
\text { Group 2012) }\end{array}$ \\
\hline
\end{tabular}

It is important to note that Aggregation and Specialization of Objects cannot directly be used for further model-driven development of systems, since additional information needs to be added by IS experts, like for example whether the Specialization between Objects is total or not. CHOOSE is not intended to be directly used for implementation (e.g., to build an enterprise database for the SME), but rather a means to provide an EA overview for the SME's CEO or managers. As such, it could be a starting point for further detailed elaboration and analysis.

\subsection{CHOOSE Constraints Using OCL}

Finally, the metamodel is completed by adding constraints (see Appendix 2: Table 3 for a full list of the constraints). These constraints are meta-constraints as they constrain metamodel components. They are to be determined at metamodel definition time, checked at model-building time when enough model elements are available in each view, and rechecked at model evolution time when the linked items are changed. Most rule-based checks can be fully automated through queries on a model database structured according to the metamodel, for instance in further software tool development efforts (Bernaert et al. 2013a; Dumeez et al. 2013; Ingelbeen et al. 2013). The main advantage of having constraints is that constraint violations drive models towards structural consistency. Further, since missing items are often revealed, these constraints address structural completeness as well (Paige et al. 2007). Next to these universal consistency rules, a model may also be further constrained by business-specific rules at M1-level. 
Within the constraints, a distinction can be made between hard or soft ones, on the one hand, and intra-view or inter-view ones, on the other hand. Hard constraints (1, 4, 13-20, 25-26) refer to those that must never be violated, while soft constraints $(2-3,5-12,21-24)$ can be seen as recommendations for the SME in order to arrive at a more balanced and complete enterprise model. The latter make it possible for the user to figure out what remains to be done at any step of the model-building method. The distinction between intra-view and inter-view constraints involves the extent to which the whole model is checked or not. Intra-view constraints (1-2, 13-20, 25-26) are related to only one of the four viewpoints of CHOOSE and are marked by the corresponding colour. They check the structural consistency, completeness, and correctness within just one of the views. Inter-view constraints $(3-12,21-24)$, in contrast, are related to at least two of the four viewpoints and are also marked by the corresponding colours. These constraints are not limited to just one viewpoint, but check the structural consistency, completeness, and correctness of the whole model. As mentioned before, this improves the cohesion of the four viewpoints and enhances the integration and traceability of the different domains of a company.

Some constraints $(2-3,5,7-8,10-12,21,23-24,26)$ are based on earlier KAOS constraints (Van Lamsweerde 2009), but often required some alterations because of the changed metamodel, as mentioned earlier (e.g., Actors can be linked to more Goals, Operations can be performed by more Actors and can also be linked to non-leaf Goals). Some new constraints (1, 4, 6, 9, 13-20, 22, 25) had to be developed since the KAOS metamodel was adapted to form the basis of the CHOOSE metamodel and inconsistent or incomplete models were discovered during the action research.

The constraints are expressed using the object constraint language (OCL), a standard of OMG (2012a) that can easily be used with the other OMG standards UML and SBVR (Warmer and Kleppe 2003). In order for the constraints to be tested and validated on instantiations of the metamodel, a UML-based specification environment tool (USE) was used, which was developed to test OCL constraints on UML models (Gogolla et al. 2007). Of course, this was not presented to the SMEs, since this software tool is rather meant to support the CHOOSE metamodel development effort and is not adapted to the characteristics of SMEs and EA. In Table 3 of Appendix 2 the metamodel including a full list of all constraints is presented as the text file serving as input for the USE tool. An example of resolving a constraint violation is given in Fig. 10 of section 8.1 .

Although this set of constraints proved to be sufficient for developing the EA model of the action research SME, it can definitely be extended. A possible future area of research could involve other relevant constraints and queries, for example to assist in conflict management (van Lamsweerde et al. 2002) or reasoning about alternative options (Heyse et al. 2012; Mylopoulos et al. 1992).

\subsection{Model Viewpoints}

It became clear during the action research that even though the CHOOSE metamodel contains few elements, the CHOOSE models became quite large, even in small SMEs (see Fig. 8). 


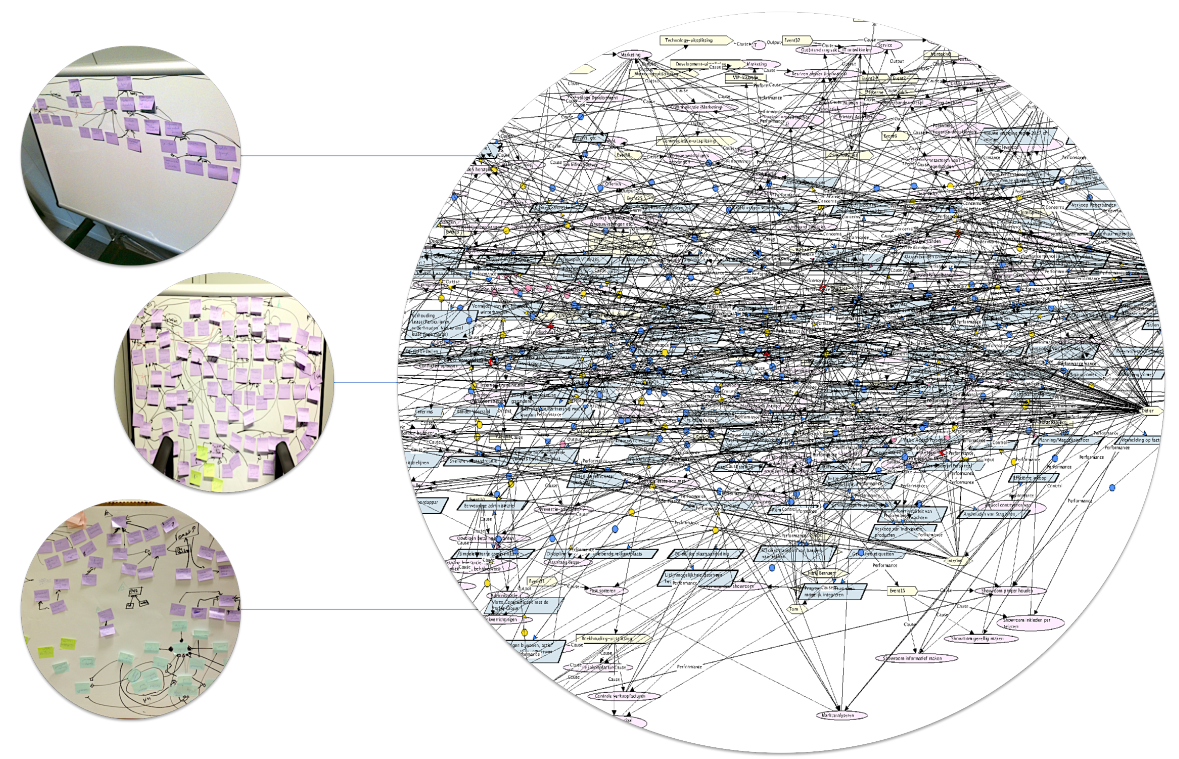

Fig. 8 The CHOOSE model of the action research SME (using post-its and afterwards inserting it in the Objectiver tool for KAOS) became quite large

Therefore, queries can be used on the model database to extract other model views and visualize these for dedicated analyses, in order to be able to keep an overview of the EA model. For instance, if an Object is Output of an Operation under the Performance of an Actor and Input for an Operation under the performance of another Actor, an implicit relationship exists between the Actors since they are dependent on each other. This provides a useful, direct view of mutual interfaces among Actors. Another example is load analysis, to see what Operations each Actor is Performing, or a RACI chart, if the Performance Types are according to RACI. Problematic situations can be spotted where a Human Actor appears overloaded.

A sufficient set of viewpoints will be further developed with the help of additional case studies.

\section{CHOOSE Metamodel Evaluation}

The evolving CHOOSE metamodel was evaluated through the different rounds of the action research programme. The results of this evaluation will be summarized in this section, and it will be determined whether the final metamodel supports the essential EA dimensions (section 4.2) and meets the requirements for EA for SMEs (section 2.2).

\subsection{Action Research Evaluation and Example}

The action research effort demonstrated that CHOOSE enables the development and management of an EA model for SMEs. It made the CEO think about his SME, how things work, why things are done, who is involved in and responsible for what, what the conflicting goals of different stakeholders are, and how balanced decisions should be made between these conflicting goals. In this respect, one specific advantage was that the CEO of the action research SME became able to assess which operations could be executed by software instead of by the employees that executed them up to that moment. For example, because of some insights from the CHOOSE model, the CEO decided to purchase an extra module for the ERP system. This module allowed him to automatically link payments with the correct customer, an operation that he used to have to do himself and that was very time-consuming. 
In general, it is safe to say that the CHOOSE model enabled a better control of the SME, with improved communication and interaction, by offering a holistic overview, in which elements are part of a bigger picture. The approach was primarily used in a top-down manner (i.e. from Goals to Operations), thus increasing the CEO's control of the SME. At the same time, though, CHOOSE also increased communication and interaction among employees and other stakeholders, as it was also used to discuss parts of the model with them. Although the terminology may not be clear to all users right now, this will definitely be remedied by the software tools we are developing (Bernaert et al. 2013a; Dumeez et al. 2013; Ingelbeen et al. 2013). A final advantage could be that employees may become more motivated if they know how their role is situated within the bigger picture of the whole SME. This was not yet visible in the SME, but longer-term evaluation will undoubtedly provide more insight into this type of benefits.

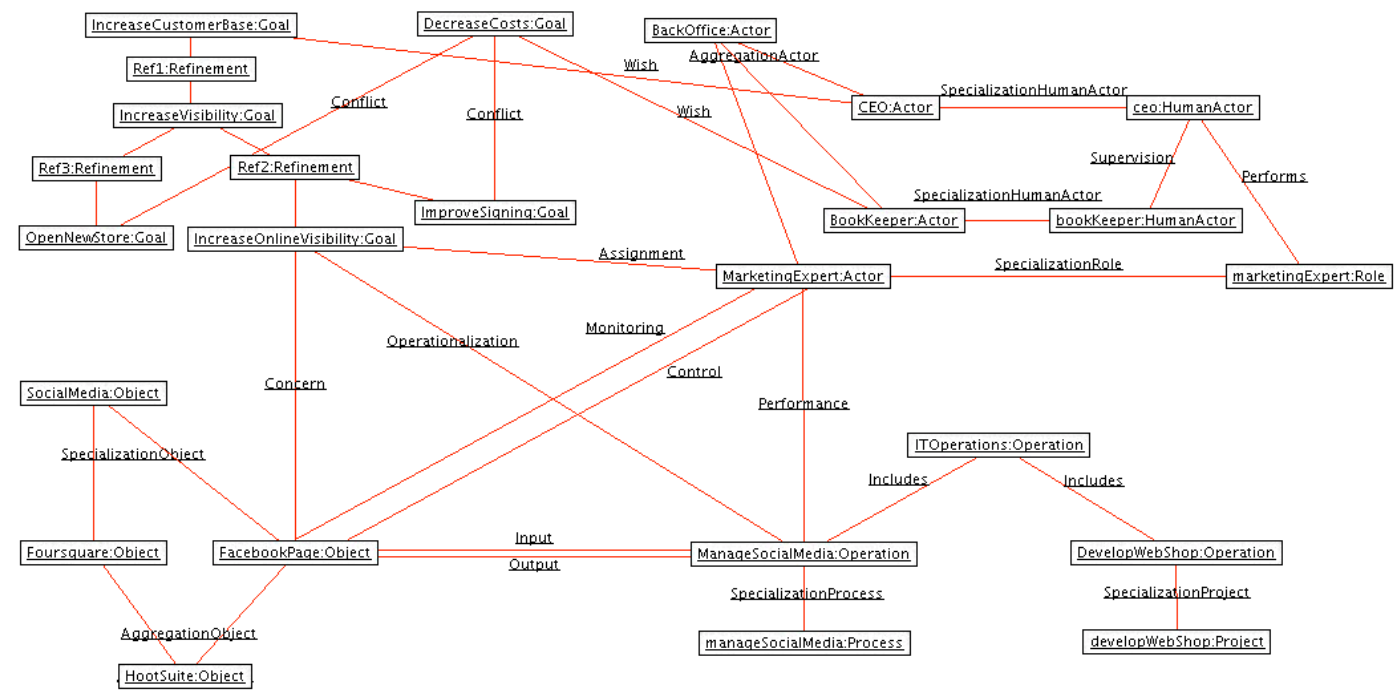

Fig. 9 Extract from the CHOOSE model from the action research SME

In order to illustrate most of CHOOSE's concepts and relationships, Fig. 9 shows an extract from the SME's CHOOSE model, modelled in the USE tool. In this example from the action research SME, the CEO wished to increase the customer base by increasing visibility in one of two possible ways. First, he could open a new store, but this conflicts with decreasing the costs, an objective of the bookkeeper. Second, he could improve the signage of the building and enhance online visibility. This second alternative was chosen. Since signage can be a pricy affair and thus conflicts with decreasing the cost, first online visibility was enhanced. In this particular SME, the CEO also performs the role of a marketing expert and is part of the SME's back office together with the bookkeeper, who is supervised by the CEO. As marketing expert, the CEO is assigned to the goal of increasing the online visibility. This is operationalized by managing the social media. More specifically, the SME's Facebook page will be managed and the marketing expert can see (i.e. Monitor) and even change (i.e. Control) this page. Managing social media is part of the IT operations in this SME, like for example also the project of the web shop development. The company also has a Foursquare page as a kind of social media, however, nothing is currently being done with this page. Both Facebook and Foursquare are part of the SME's Hootsuite account, in which different social media platforms can be managed. 

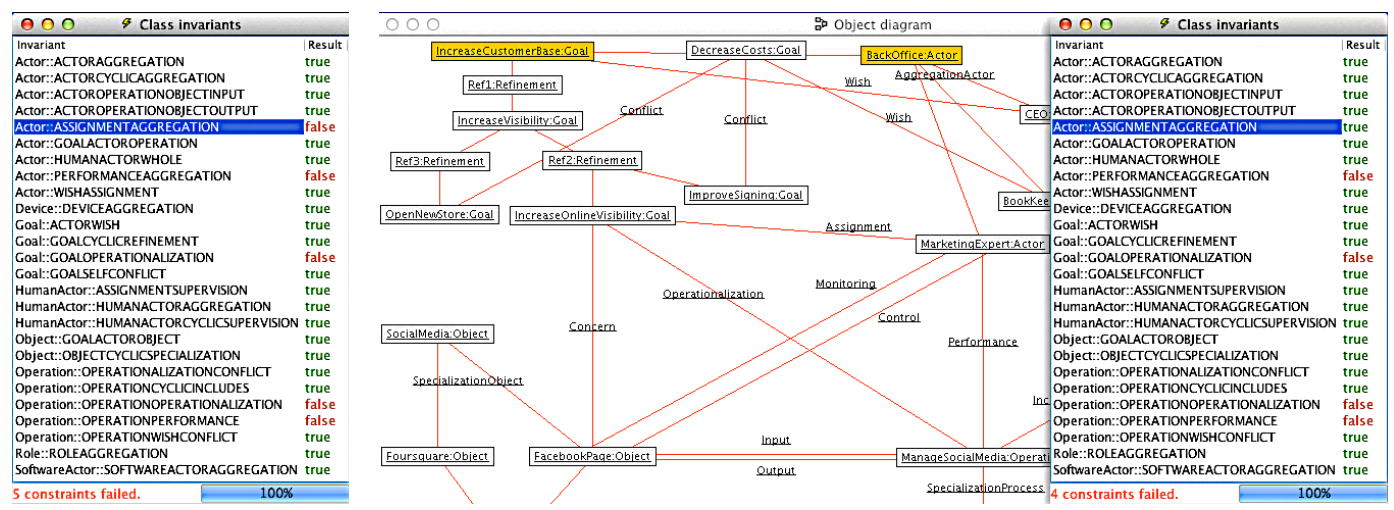

Fig. 10 OCL constraints failed (left) and one constraint resolved (right)

Five OCL constraints are violated in this model extract (left part of Fig. 10), which could guide the SME to make the CHOOSE model more consistent and complete. For example, ASSIGNMENTAGGREGATION (constraint 5) failed because the Actor BackOffice has no Assignment relation with any of the higher-level Goals of IncreaseOnlineVisibility, which is Assigned to MarketingExpert, one of the subActors of BackOffice. This could be resolved by Assigning BackOffice to IncreaseCustomerBase (right part of Fig. 10)

\subsection{Support of Essential EA Dimensions}

The CHOOSE metamodel conforms to the essential dimensions of EA frameworks as identified after analysis of important EA frameworks in section 4:

- The CHOOSE metamodel covers and integrates the four essential EA focuses: why through the goal viewpoint, who through the actor viewpoint, how through the operation viewpoint, and what through the object viewpoint. Relationships are defined to relate concepts from different viewpoints.

- The CHOOSE metamodel blends the three EA layers (business, IS, IT) by providing Actors for each layer (Human Actor / Role, Software Actor, Device) and enabling the other three viewpoints to be related to it. Goals, Operations, and Objects could also originate from the three different EA layers, as seen in the EA model of the SME during the action research. Yet, for this SME, no explicit specialization was needed.

- The CHOOSE metamodel provides a means to analyze the strategy space without worrying about any constraints of operations beforehand, as it separates Goals from Operations via Operationalization links.

\subsection{Meeting the Requirements for EA for SMEs}

The CHOOSE metamodel conforms to the EA requirements listed in section 2.2.1:

1. By providing a means to analyze the SME by using a metamodel, control was increased for the CEO. Constraints in OCL that are generally applicable are presented. SME-specific queries can be made on the EA model.

2. By conforming to the essential parts of EA frameworks, a holistic overview can be provided, but the SME is not obliged to make a global model. If necessary, the models can be made for one project at a time (Ross et al. 2006). The when and where-focus can be considered to be part of the operation viewpoint, which could be elaborated by business process modelling 
languages. The SME did not need separate Operation attributes for these two focuses, since the Description attribute was sufficient to describe details.

3. Since the CHOOSE metamodel is based on goal refinements, the requirement regarding objectives is fulfilled.

4. This requirement (fit for the target audience) was split up into SME-specific requirements (see further).

5. Since CHOOSE is based on the essential dimensions from EA frameworks used for modelling enterprises, it provides an enterprise overview.

As the fourth requirement of EA is related to SMEs as a target audience (and, more specifically, to the CEOs or managers of SMEs), the requirements for adoption and successful use of IT in SMEs can be discussed (section 2.2.2):

4.1. To allow the CEO to work more efficiently, the CHOOSE metamodel is kept to the bare minimum (e.g., a comparison can be made between the number of metamodel elements and relationships in CHOOSE and ArchiMate). Nevertheless, a metamodel by itself did not appear to offer the CEO enough flexibility to work whenever and wherever he had the time for it. Further software tool support (i.e. research step 5 in Fig. 1) should developed to make this possible (Ernst et al. 2006). In the fourth round of the action research programme, a prototype CHOOSE software tool was installed in this SME.

4.2. To make the approach accessible to people with few IT or modelling skills, the metamodel is kept as simple as possible (including some optional parts that do not have to be used), with just four viewpoints that each contain only one central concept. The CEO was able to work with CHOOSE and is now also using the software tool. Still, a longer-term evaluation and further case studies are needed to improve the CHOOSE approach and software tool support.

4.3. Throughout the different rounds of the action research programme, the researchers guided the CEO in the development of the EA model. After the fourth round, the CEO started working with the software tool himself. The ultimate goal is to further develop the CHOOSE approach so that any need for external help is reduced to a minimum.

4.4. A process overview can be built with the operation viewpoint. Processes (or projects) can be elaborated by using a business process management approach (or project management approach) and linking this to the corresponding process (or project) in the CHOOSE model. In the SME of the action research, no processes were elaborated. As this could be the case in other SMEs, further research is still needed on how to easily link process models to the process overview (e.g., by providing attachment options in the software tool support).

4.5. The CEO was involved in developing the CHOOSE model, as he possessed the required knowledge to make an overview of the SME. The CHOOSE model is an instantiation of the CHOOSE metamodel that is developed and further refined throughout the action research cycles, based on the problems the CEO and the researchers encountered.

4.6. In terms of complexity, the number of metamodel concepts and relationships of CHOOSE is considerably lower than in other EA frameworks and kept to the bare minimum. The main benefits in the SME from the action research were threefold. First, because the EA was built from scratch, this offered considerable insight into the structure and inner workings of the 
SME. It was clear that the CEO became very enthusiastic after he had explicated his goals for the SME, because he experienced this entire process as a steep learning curve. Second, when managing the EA, the CHOOSE metamodel helped store decisions of meetings in one place (i.e. in the EA model). Third, CHOOSE provided the SME a platform for analysis and guided change, especially because of the built-in traceability by integrating four viewpoints into one metamodel. Among other things, it became possible to predefine analyses and enabled easy querying. In the SME, it was the OCL constraints that gave the most guidance. However, additional benefits are now becoming apparent while the SME is actually using the software tool. Some functions have already been programmed (e.g., Excel output, different viewpoints, querying) and others will mainly be developed in line with the feedback from the case study research that is conducted in other SMEs.

\section{Conclusion}

This paper presents the design of the CHOOSE metamodel as the first effort to develop an EA approach specifically tailored to SMEs. The CHOOSE metamodel is designed according to the requirements for EA in an SME context (Bernaert et al. 2013b). This is achieved by means of an action research programme in one specific SME, complemented by case study research in five more SMEs. The resulting metamodel is expressed as a UML class diagram, and extended with concept and relationship definitions in SBVR and intra- and inter-view constraints in OCL.

As the action research SME implemented certain changes according to the insights gained from the EA model, it was clear that the CHOOSE metamodel was indeed very valuable. In fact, CHOOSE is still used in the SME, with the help of a software tool to support it.

Nevertheless, further work is still required. A first limitation is that the scope of the research was limited to a single company, which is typical of action research. However, five more case studies were concurrently performed in different kinds of SMEs, as research indicates that SMEs differ significantly in size, sector, and other factors (Bernaert et al. 2013b). The input, management, and output of CHOOSE models are hence tested in multiple SME settings. These case studies serve as input for the development and refinement of a method with step-by-step guidelines, for a further evaluation of the metamodel presented in this paper, and for the evaluation of the benefits of EA for SMEs. For example, the explicit representation of business rules in the CHOOSE metamodel has to be further examined if the need arises in further case studies. Possibilities of how this representation could be achieved can be found in (Businska et al. 2012).

Another area for future research involves software tool support for different platforms. This would enable an easier interface for SMEs to input, adjust, and analyze their EA model. Prototypes for PCs (Ingelbeen et al. 2013), smartphones, and tablets (Bernaert et al. 2013a; Dumeez et al. 2013) have already been developed and are currently being tested in different case studies. At present, we are also working on different possibilities to make as-is and to-be models and analyses, and are testing which best meet the needs of the SMEs. Moreover, the cognitive effectiveness of alternative notations for CHOOSE models (Boone et al. 2014) is being researched so as to provide a more efficient and effective visualization, since this also influences usability (Henderson-Sellers et al. 2012; Moody 2009). Finally, an integration with ArchiMate is being 
developed. This would allow users to switch from CHOOSE to ArchiMate if a more elaborate EA approach is needed to increase effectiveness for experienced EA users (Rossi and Brinkkemper 1996) (e.g., if a more detailed representation of the IT architecture would be needed), or to switch from ArchiMate to CHOOSE (Roose et al. 2013).

\section{References}

Aarabi, M., Saman, M. Z. M., Wong, K. Y., Beheshti, H. M., \& Hemdi, A. R. The Effect of Enterprise Architecture for Enterprise Resource Planning in Small and Medium Enterprises: a Review and Research Direction. In IESS, Indonesia, 2011 (pp. 159-163)

Andersson, B., Johannesson, P., \& Zdravkovic, J. (2009). Aligning Goals and Services through Goal and Business Modelling. Inf Syst E-Bus Manage, 7(2), 143-169.

Anton, A. I. Goal-Based Requirements Analysis. In ICRE, 1996 (pp. 136-144)

Anton, A. I., McCracken, W. M., \& Potts, C. Goal Decomposition and Scenario Analysis in Business Process Reengineering. In CAiSE, 1994 (Vol. 811, pp. 94-104, LNCS): Springer

Balabko, P., \& Wegmann, A. (2006). Systemic classification of concern-based design methods in the context of enterprise architecture. Inf Syst Front, 8(2), 115-131.

Baskerville, R., \& Myers, M. D. (2004). Special Issue on Action Research in Information Systems: Making IS Research Relevant to Practice: Foreword. MIS Q, 28(3), 329-335.

Bernaert, M., Maes, J., \& Poels, G. (2013a). An Android Tablet Tool for Enterprise Architecture Modeling in Small and Medium-Sized Enterprises. In The Practice of Enterprise Modeling (pp. 145-160): Springer.

Bernaert, M., \& Poels, G. The Quest for Know-How, Know-Why, Know-What and Know-Who: Using KAOS for Enterprise Modelling. In CAiSE International Workshops, 2011 (Vol. 83, pp. 29-40, LNBIP): Springer

Bernaert, M., Poels, G., Snoeck, M., \& De Backer, M. (2013b). Enterprise Architecture for Small and Medium-Sized Enterprises: A Starting Point for Bringing EA to SMEs, Based on Adoption Models. In Information systems and small and medium-sized enterprises (SMEs) : state of art of IS research in SMEs: Springer.

Bhagwat, R., \& Sharma, M. K. (2007). Information system architecture: a framework for a cluster of smalland medium-sized enterprises (SMEs). Prod Plan Control, 18(4), 283-296.

Bidan, M., Rowe, F., \& Truex, D. (2012). An empirical study of IS architectures in French SMEs: integration approaches. Eur J Inf Syst, 21(3), 287-302.

Bittler, R. S., \& Kreizmann, G. (2005). Gartner Enterprise Architecture Process. Evolution, 21.

Boman, M., Bubenko, J. A., Johannesson, P., \& Wangler, B. (1997). Conceptual modelling: Prentice-Hall, Inc.

Boone, S., Bernaert, M., Roelens, B., Mertens, S., \& Poels, G. (2014). Evaluating and Improving the Visualisation of CHOOSE, an Enterprise Architecture Approach for SMEs. In The Practice of Enterprise Modeling (Vol. 197, pp. 87-102, LNBIP): Springer Berlin Heidelberg.

Braun, C., \& Winter, R. A Comprehensive Enterprise Architecture Metamodel and Its Implementation Using a Metamodeling Platform. In EMISA, 2005 (pp. 64-79)

Bubenko, J. Extending the Scope of Information Modelling. In DAISD, 1993 (pp. 73-97)

Business Transformation Agency (2009). Vocabulary-Driven Enterprise Architecture Development: Guidelines for DoDAF AV-2: Design and Development of the Integrated Dictionary.

Businska, L., Kirikova, M., Penicina, L., Buksa, I., \& Rudzajs, P. Enterprise Modeling for Respecting Regulations. In PoEM, 2012 (pp. 106-117)

Clark, T., Barn, B. S., \& Oussena, S. LEAP: A Precise Lightweight Framework for Enterprise Architecture. In ISEC, 2011 (pp. 85-94): ACM

Daneva, M., \& van Eck, P. (2007). What Enterprise Architecture and Enterprise Systems Usage Can and Can not Tell about Each Other. Int J Comput Sci Appl, 4, 93 - 109.

Dardenne, A., Fickas, S., \& van Lamsweerde, A. Goal-directed Concept Acquisition in Requirements Elicitation. In IWSSD, 1991 (pp. 14-21): IEEE

Dardenne, A., van Lamsweerde, A., \& Fickas, S. (1993). Goal-directed requirements acquisition. Sci Comput Program, 20(1-2), 3-50.

Database Systems Group (2013). USE: A UML based Specification Environment. http://sourceforge.net/projects/useocl/.

Denzin, N. K. (2006). Sociological Methods: A Sourcebook: Transaction Publishers.

Devos, J. (2011). IT Governance for SMEs. University of Ghent, Ghent.

DoD (2010). DoDAF Architecture Framework Version 2.2. http://dodcio.defense.gov/dodaf20.aspx.

Dumeez, J., Bernaert, M., \& Poels, G. Development of Software Tool Support for Enterprise Architecture in Small and Medium-Sized Enterprises. In CAiSE International Workshops, 2013 (LNBIP): Springer

Engelsman, W., Quartel, D., Jonkers, H., \& van Sinderen, M. (2011). Extending enterprise architecture modelling with business goals and requirements. Enterp Inf Syst, 5(1), 9-36. 
Engelsman, W., \& Wieringa, R. (2012). Goal-Oriented Requirements Engineering and Enterprise Architecture: Two Case Studies and Some Lessons Learned. In REFSQ (Vol. 7195, pp. 306-320, LNCS): Springer.

Erickson, J., \& Siau, K. Can UML Be Simplified? Practitioner Use of UML in Separate Domains. In EMMSAD, 2007 (pp. 89-98): Citeseer

Eriksson, H.-E., \& Penker, M. (2000). Business Modeling with UML: Business Patterns at Work: Wiley.

Ernst, A. M., Lankes, J., Schweda, C. M., \& Wittenburg, A. Tool Support for Enterprise Architecture Management - Strengths and Weaknesses. In EDOC, 2006 (pp. 13-22)

European Commission (2011). Are EU SMEs recovering from the crisis? Annual Report on EU Small and Medium sized Enterprises 2010/2011.

French, W. L., \& Bell, C. H. (1973). Organization Development: Behavioral Science Interventions for Organization Improvement: Prentice-Hall.

Gartner (2012). Gartner's 2011 Global Enterprise Architecture Survey: EA Frameworks Are Still Homemade and Hybrid.

Georgiadis, G. (2015). Development of an ontology and risk-led method for selecting and tailoring Enterprise Architecture Frameworks. In W. Paper (Ed.): Working Paper, Ghent University.

Glissman, S., \& Sanz, J. (2009). A Comparative Review of Business Architecture. IBM.

Gogolla, M., Büttner, F., \& Richters, M. (2007). USE: A UML-based specification environment for validating UML and OCL. Sci Comput Program, 69(1-3), 27-34.

Hannon, P. D., \& Atherton, A. (1998). Small firm success and the art of orienteering: the value of plans, planning, and strategic awareness in the competitive small firm. J Small Bus Enterp Dev, 5(2), 102119.

Hegge, H. M. H., \& Wortmann, J. C. (1991). Generic bill-of-material: a new product model. Int J Prod Econ, 23(1-3), 117-128.

Henderson, J. C., \& Venkatraman, N. (1993). Strategic Alignment: Leveraging Information Technology for Transforming Organizations. IBM Syst J, 32(1), 4-16.

Henderson-Sellers, B., Low, G., \& Gonzalez-Perez, C. Semiotic Considerations for the Design of an AgentOriented Modelling Language. In EMMSAD, 2012 (Vol. 113, pp. 422-434): Springer LNBIP

Hevner, A. R., March, S. T., Park, J., \& Ram, S. (2004). Design Science in Information Systems Research. MIS Q, 28(1), 75-105.

Heyse, M., Bernaert, M., \& Poels, G. (2012). Keuzes Maken binnen Processen: Het Vermijden van een Russische Roulette voor de Organisaties. University of Ghent, Ghent.

Hoogervorst, J. (2004). Enterprise Architecture: Enabling Integration, Agility and Change. Int J Coop Info Syst, 13(3), 213-233.

IEEE Computer Society (2000). IEEE Recommended Practice for Architectural Description of SoftwareIntensive Systems. IEEE Std 1471-2000.

IFEAD (2005). Trends in Enterprise Architecture 2005: How are Organizations Progressing?

IFEAD (2006). Extended Enterprise Architecture Framework Essentials Guide.

Ingelbeen, D., Bernaert, M., \& Poels, G. Enterprise Architecture Software Tool Support for Small and Medium-Sized Enterprises: EASE. In AMCIS, 2013

ISACA (2012). COBIT 5. http://www.isaca.org/COBIT/Pages/COBIT-5-Framework-productpage.aspx

Jacobs, D., Kotzé, P., van der Merwe, A., \& Gerber, A. Enterprise Architecture for Small and Medium Enterprise Growth. In EEWC, 2011 (Vol. 79, pp. 61-75, LNBIP): Springer

James, G. A., Handler, R. A., Lapkin, A., \& Gall, N. (2005). Gartner Enterprise Architecture Framework. Evolution.

Järvinen, P. (2007). Action Research is Similar to Design Science. Qual \& Quant, 41(1), 37-54.

Jonkers, H., Lankhorst, M. M., ter Doest, H. W. L., Arbab, F., Bosma, H., \& Wieringa, R. J. (2006). Enterprise architecture: Management tool and blueprint for the organisation. Inf Syst Front, 8(2), 63-66.

Kerzner, H. R. (2013). Project Management: A Systems Approach to Planning, Scheduling, and Controlling (Vol. 11): Wiley.

Kroon, B., Voorde, K., \& Timmers, J. (2012). High performance work practices in small firms: a resourcepoverty and strategic decision-making perspective. Small Bus Econ, 1-21.

Lankhorst, M. (2013). Enterprise Architecture at Work: Modelling, Communication and Analysis (Vol. 3). New York: Springer.

Leist, S., \& Zellner, G. Evaluation of Current Architecture Frameworks. In SAC, 2006 (pp. 1546-1553): ACM

Letier, E., \& van Lamsweerde, A. Deriving Operational Software Specifications from System Goals. In SIGSOFT, 2002 (pp. 119-128): ACM

Letier, E., \& van Lamsweerde, A. Reasoning about Partial Goal Satisfaction for Requirements and Design Engineering. In SIGSOFT, 2004 (pp. 53-62): ACM

Lindström, A., Johnson, P., Johansson, E., Ekstedt, M., \& Simonsson, M. (2006). A survey on CIO concernsdo enterprise architecture frameworks support them? Inf Syst Front, 8(2), 81-90.

Loucopoulos, P., \& Kavakli, E. (1995). Enterprise Modelling and the Teleological Approach to Requirements Engineering. Int J Coop Inf Syst, 4(1), 45-79.

Luftman, J., \& Ben-Zvi, T. (2011). Key Issues for IT Executives 2011: Cautious Optimism in Uncertain Economic Times. MIS Q Exec, 10(4), 203-212. 
Lybaert, N. (1998). The Information Use in a SME: Its Importance and Some Elements of Influence. Small Bus Econ, 10(2), 171-191.

Moody, D. (2009). The Physics of Notations: Toward a Scientific Basis for Constructing Visual Notations in Software Engineering. IEEE Trans Softw Eng, 35(6), 756-779.

Moody, D. L. The Method Evaluation Model: A Theoretical Model for Validating Information Systems Design Methods. In ECIS, 2003

Mostow, J. (1985). Towards Better Models Of The Design Process. AI Mag, 6(1), 44-57.

Mylopoulos, J., Chung, L., \& Nixon, B. (1992). Representing and Using Nonfunctional Requirements: A Process-Oriented Approach. IEEE T Softw Eng, 18(6), 483-497.

O'Regan, N., \& Ghobadian, A. (2004). The importance of capabilities for strategic direction and performance. Manag Decis, 42(2), 292-313.

OMG (2008). Semantics of Business Vocabulary and Business Rules (SBVR) (v1.0). http://www.omg.org/spec/SBVR/1.0/.

OMG (2009). Organization Structure Metamodel (OSM). http://www.omg.org/cgi-bin/doc?bmi/09-0802 .

OMG (2010). Business Motivation Model (BMM) (v1.1). http://www.omg.org/spec/BMM/1.1/.

OMG (2011a). Business Process Model and Notation (BPMN) (v2.0). http://www.omg.org/spec/BPMN/2.0/.

OMG (2011b). OMG Unified Modeling Language (OMG UML), Infrastructure (v2.4.1). http://www.omg.org/spec/UML/2.4.1/.

OMG (2011c). OMG Unified Modeling Language (OMG UML), Superstructure (v2.4.1). http://www.omg.org/spec/UML/2.4.1/.

OMG (2012a). OMG Object Constraint Language (OCL) (v2.3.1). http://www.omg.org/spec/OCL/2.3.1/.

OMG (2012b). OMG's MetaObject Facility. http://www.omg.org/mof/.

OMG (2013). MDA - The Architecture Of Choice For A Changing World. http://www.omg.org/mda/.

Paige, R. F., Brooke, P. J., \& Ostroff, J. S. (2007). Metamodel-Based Model Conformance and Multiview Consistency Checking. ACM Trans Softw Eng Methodol, 16(3).

Project Management Institute (2013). Project Management Body Of Knowlegde (PMBOK). http://www.pmi.org/PMBOK-Guide-and-Standards.aspx.

Radeke, F. Toward Understanding Enterprise Architecture Management's Role in Strategic Change: Antecedents, Processes, Outcomes. In WI, 2011 (Vol. 2011)

Rescher, N. (1977). Methodological Pragmatism: Systems-Theoretic Approach to the Theory of Knowledge: Blackwell Publishers.

Respect-IT (2007). A KAOS Tutorial. http://www.respect-it.be/.

Roose, D., Vansteenlandt, J., Bernaert, M., \& Poels, G. (2013). Development of a common base for enterprise architecture: building the bridge between CHOOSE and ArchiMate. University of Ghent, Ghent.

Ross, J. W., Weill, P., \& Robertson, D. (2006). Enterprise Architecture as Strategy: Creating a Foundation for Business Execution: Harvard Business Review Press.

Rossi, M., \& Brinkkemper, S. (1996). Complexity Metrics for Systems Development Methods and Techniques. Inf Syst, 21(2), 209-227.

Sandkuhl, K., Stirna, J., Persson, A., \& Wißotzki, M. (2014). Enterprise Modeling: Tackling Business Challenges with the 4EM Method: Springer.

Scheer, A.-W. (2000). ARIS - Business Process Modeling (Vol. 3): Springer.

Schekkerman, J. (2006). How to Survive in the Jungle of Enterprise Architecture Frameworks: Creating or Choosing an Enterprise Architecture Framework: Trafford Publishing.

Sessions, R. (2007). Comparison of the Top Four Enterprise Architecture Methodologies. Microsoft.

Small Business Administration (2011). How Important are Small Businesses to the U.S. Economy? http://www.sba.gov/advo.

Smith, J. A. (1998). Strategies for Start-Ups. Long Range Plan, 31(6), 857-872.

Snoeck, M., Dedene, G., Verhelst, M., \& Depuydt, A.-M. (1999). Object-Oriented Enterprise Modelling with $M E R O D E$ : Leuven University Press.

Stirna, J., \& Persson, A. Ten Years Plus with EKD: Reflections from Using an Enterprise Modeling Method in Practice. In EMMSAD, 2007 (pp. 99-108)

Susman, G. I., \& Evered, R. D. (1978). An Assessment of the Scientific Merits of Action Research. Admin Sci Q, 23(4), 582-603.

Tamm, T., Seddon, P. B., Shanks, G., \& Reynolds, P. (2011). How Does Enterprise Architecture Add Value to Organisations? CAIS, 28(1).

The Open Group (2009). TOGAF Version 9. http://www.opengroup.org/togaf.

The Open Group (2012). ArchiMate http://www3.opengroup.org/subjectareas/enterprise/archimate.

The White House OMB (2012). The Common Approach To Federal Enterprise Architecture.

The White House OMB (2013). Federal Enterprise Architecture. http://www.whitehouse.gov/omb/egov/FEA.

Urbaczewski, L., \& Mrdalj, S. (2006). A Comparison of Enterprise Architecture Frameworks. Issues Inf Syst, $7(2), 18-23$

van 't Wout, J., Waage, M., Hartman, H., Stahlecker, M., \& Hofman, A. (2010). The Integrated Architecture Framework Explained: Why, What, How: Springer. 
Van Lamsweerde, A. (2009). Requirements Engineering: From System Goals to UML Models to Software Specifications (Vol. 3): Wiley.

van Lamsweerde, A., Dardenne, A., Delcourt, B., \& Dubisy, F. The KAOS Project: Knowledge Acquisition in Automated Specification of Software. In AAAI Spring Symposium Series, Stanford University, 1991 (pp. 59-62)

van Lamsweerde, A., Darimont, R., \& Letier, E. (2002). Managing Conflicts in Goal-Driven Requirements Engineering. IEEE Trans Softw Eng, 24(11), 908-926.

van Lamsweerde, A., Darimont, R., \& Massonet, P. Goal-Directed Elaboration of Requirements for a Meeting Scheduler: Problems and Lessons Learnt. In RE, 1995 (pp. 194-203): IEEE

Veasey, P. W. (2001). Use of enterprise architectures in managing strategic change. Bus Process Manag $J$, $7(5), 420-436$.

Wagter, R., van den Berg, M., Luijpers, J., \& van Steenbergen, M. (2005). Dynamic Enterprise Architecture: How to Make It Work: Wiley.

Warmer, J., \& Kleppe, A. (2003). The Object Constraint Language: Getting Your Models Ready for MDA (Vol. 2): Addison-Wesley Professional.

Wegmann, A., Regev, G., Rychkova, I., Lê, L.-S., de la Cruz, J. D., \& Julia, P. Business and IT Alignment with SEAM for Enterprise Architecture. In EDOC, 2007

Weske, M. (2012). Business Process Management: Concepts, Languages, Architectures (Vol. 2): Springer.

Winter, R., \& Fischer, R. (2007). Essential Layers, Artifacts, and Dependencies of Enterprise Architecture. $J$ Enterp Archit, 3(2), 7-18

Wißotzki, M., \& Sonnenberger, A. Enterprise Architecture Management - State of Research Analysis \& a Comparison of Selected Approaches. In PoEM, 2012 (pp. 37)

Yu, E. S. K. Modelling Organizations for Information Systems Requirements Engineering. In RE, 1993 (pp. 34-41): IEEE

Zach, O. (2012). ERP System Implementation in Small and Medium-Sized Enterprises. University of Agder, Agder.

Zachman International (2011). Zachman Version 3.0. http://www.zachman.com.

Zachman, J. A. (1987). A framework for information systems architecture. IBM Syst J, 26(3), 276-292. 


\section{Appendix 1: KAOS Metamodel}

Each viewpoint is discussed separately and then the integrated KAOS metamodel is presented in Fig. 11. Concepts from the goal, agent, operation, object, and behaviour viewpoints will be respectively coloured in yellow, red, purple, green, and grey. Attributes between square brackets are optional attributes.

\section{KAOS Goal Viewpoint}

The central element of the KAOS goal viewpoint is a Goal. A Goal is a prescriptive statement of intent that the system should satisfy through the cooperation of its Agents. The formulation is declarative, unlike Operational procedures to implement it. A Goal can be of a specific type (SoftGoal or BehaviourGoal (Achieve or Maintain/Avoid)) and of a specific [Category] (functional or non-functional).

Goal Refinement is enabled by refining higher-level Goals in zero or more Refinements (ORRef) that group (AND-Ref) one or more lower-level Goals. An AND-Ref (OR-Ref) means that the parent Goal can be satisfied/satisficed by satisfying/satisficing all (one or more) child Goals in the Refinement. A LeafGoal is a Goal that can be under the Responsibility of exactly one Agent and is a Requirement or Expectation, depending on the type of Agent that has a Responsibility relationship with it (respectively SoftwareToBeAgent and EnvironmentAgent).

Domain properties (DomInvar) or hypotheses (DomHyp) are descriptive statements (DomDescript) holding regardless of how system Agents behave. Domain properties typically correspond to physical laws that cannot be broken.

Goals can be ObstructedBy Obstacles or can be a Resolution for Obstacles. Obstacles can be refined by $O$-Refinements in the same way as Goals can be refined by Refinements. Conflict links may interconnect Goal nodes to capture potential Conflicts among them. They are not explicitly represented in the metamodel, but are captured in the Divergence relation, which captures a potential Conflict, where some statements become logically inconsistent if a BoundaryCondition becomes true.

\section{KAOS Agent Viewpoint}

The central element of the KAOS agent viewpoint is an Agent. Agents are active system Objects that are responsible for the LeafGoals in a goal model. An Agent is responsible for a Goal by a Responsibility relationship if restricting its individual behaviour by adequate control of system items is sufficient for ensuring Goal satisfaction/satisficing.

From an operational standpoint, an Agent can be defined as a processor that performs (Performance) Operations under restricted conditions to satisfy the Goals for which it is responsible (Responsibility). For an Agent to be assigned (Assignment) to a Goal, the Goal must be realizable by the Agent in view of its capabilities. Agent capabilities are defined in terms of Object Attributes and Associations that the Agent can Monitor or Control. Monitor means that an Agent can get the values of the Attribute or can evaluate whether the Association holds, while Control means that an Agent can set values for this Attribute or can create or delete an Association. 
An Agent can be decomposed into finer-grained ones with finer-grained Responsibilities through the recursive Aggregation relationship. An Agent may be related to other Agents through Dependency links. A depender depends on a dependee for a Goal, if a dependee's failure to get this Goal satisfied/satisficed can result in a depender's failure to get one of its Assigned Goals satisfied/satisficed.

An agent model defines the boundary between the software-to-be and its environment, as an Agent can be a SoftwareToBeAgent or an EnvironmentAgent. An Agent can be of a different [Category], while this is not explicitly visible in the metamodel: NewSoftwareAgents to be developed, ExistingSoftwareAgents with which the software-to-be will have to interoperate, Devices, and HumanAgents playing specific Roles.

The Wish meta-relationship is not shown in the metamodel. It links Goal and HumanAgent and captures the fact that this HumanAgent would like the Goal to be satisfied/satisficed.

\section{KAOS Operation Viewpoint}

The operation viewpoint captures the functional services that the target system should provide in order to meet its Goals. An Operation is a binary relation over system States. Is has a tuple of Input variables and a tuple of Output variables defining its signature. An Input variable designates an Object instance to which the Operation applies. The State of this instance affects the application of the Operation. An Output variable designates an Object instance on which the Operation acts. The State of this instance is changed by the application of the Operation. An Input variable can be an Output variable for the same Operation. A particular application of the Operation yields a State Transition from a State in InputState to a State in OutputState.

An Agent performs (Performance) an Operation if the applications of this Operation are activated by instances of the Agent. Every Operation is Performed by exactly one Agent. Operationalization refers to the process of mapping LeafGoals, under the Responsibility of single Agents, to Operations ensuring them. Each such Operation is performed (Performance) by the responsible (Responsibility) Agent under restricted conditions for satisfaction/satisficing of its Goals. While a single Operation may operationalize (Operationalization) multiple Goals, a single Goal will in general be operationalized (Operationalization) by multiple Operations.

It is important to notice the difference between a Goal and an Operation. A Goal is an intentional specification: it leaves the Operations realizing it implicit, whereas an Operation is an operational specification: it leaves the intentions underlying it implicit. A Goal has a higher stability than an Operation (van Lamsweerde et al. 1995). A Goal captures an objective that the system should satisfy and is specified declaratively. An Operation captures a functional service that the system should provide to satisfy such an objective and maybe others and is specified by conditions characterizing its applicability and effect. Semantically speaking, a BehaviouralGoal constrains entire sequences of system State Transitions, while an Operation constrains single State Transitions within such sequences.

In KAOS, Operations are atomic and cannot be decomposed into finer-grained ones. Goal Refinements will be favoured, from which fine-grained Operations are derived, over Goal-free Operation refinement in an operational model (Letier and van Lamsweerde 2002). 


\section{KAOS Object Viewpoint}

The object viewpoint provides a structural view of the system and is represented by entityrelationship diagrams using the UML class diagram notation. Entities and the structural features of Events and Agents will be represented as Operation-free UML classes and Associations will be represented as UML associations. The object model gathers all concept definitions and domain properties used in the goal, agent, operation, and behaviour models and introduces a common vocabulary to refer to. The object model can later on provide a basis for generating a database schema and for elaborating a software architecture.

A conceptual Object is a discrete set of instances of a domain-specific concept that are manipulated by the modelled system. These instances are distinctly identifiable, can be enumerated in any system State, share similar features, and may differ from each other in their individual States and State Transitions. The set of instances that are members of an Object will thus generally change over time. The semantic InstanceOf relation is kept implicit in the metamodel. This built-in semantic relation allows determining which individuals are instances of the Object in the current State.

An Object can be an Association, an Entity, an Event, or an Agent. An Entity is an autonomous and passive Object. An Association is a passive Object dependent on other Objects that it Links and it is also used under the synonymous term relationship. Each Linked Object plays a specific Role in the Association. An Event is an instantaneous Object. An Agent is as already mentioned an autonomous and active Object. It is important to notice that an Agent is a subtype of an Object and inherits the relationships of an Object (Dardenne et al. 1993). An Association can Link two or more Objects, can be reflexive, can have different Multiplicities and can be a Specialization, an Aggregation, or an ApplicationSpecific type. An Association can have a Name, so a user can define different Associations with different Names. Concern links connect Goal nodes to the Objects to which they refer.

An Attribute is an intrinsic feature of an Object regardless of other Objects in the model. It has a Name and a Range of values.

\section{KAOS Behaviour Viewpoint}

The behaviour viewpoint completes the static representation of system functionalities by capturing the required system dynamics. An operation model focuses on classes of Input-Output State Transitions, an object model declares and structures the variables undergoing State Transitions, and an agent model indicates which variable is controlled by which Agent.

Since this behaviour viewpoint will not be included in the CHOOSE metamodel, it is not further explained.

\section{Integrated KAOS Metamodel and Adaptation to the CHOOSE Metamodel}

The viewpoints (excluding the behaviour viewpoint) can be combined to form the integrated KAOS metamodel (Fig. 11). The core element is each time represented in the corresponding colour. In Fig. 12, the green parts of the KAOS metamodel are the ones that were retained in the CHOOSE metamodel. Fig. 13 depicts how these elements were either used as such (green) or adapted (orange), or where new elements were added (purple) to form the CHOOSE metamodel. 


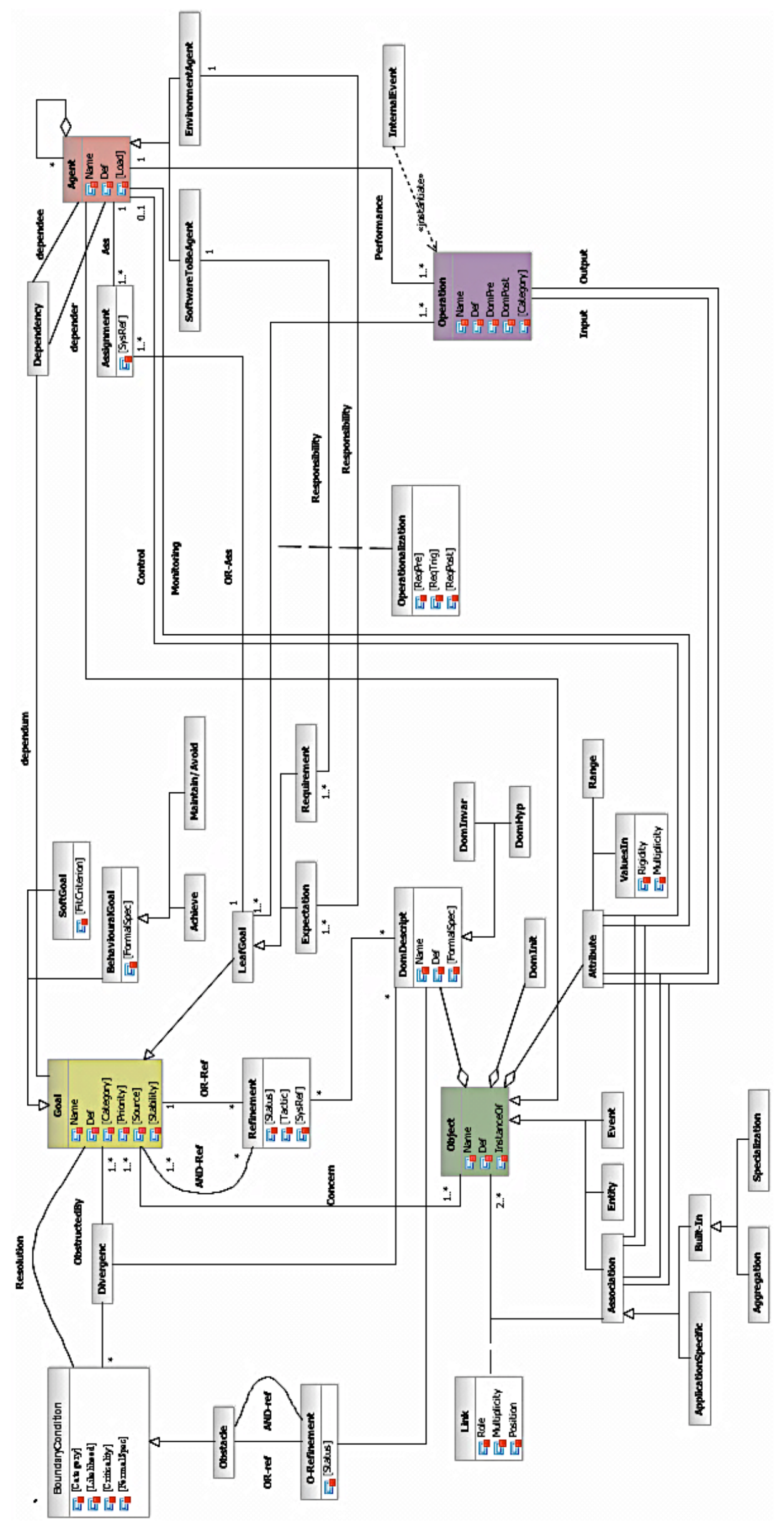

Fig. 11 KAOS integrated metamodel 


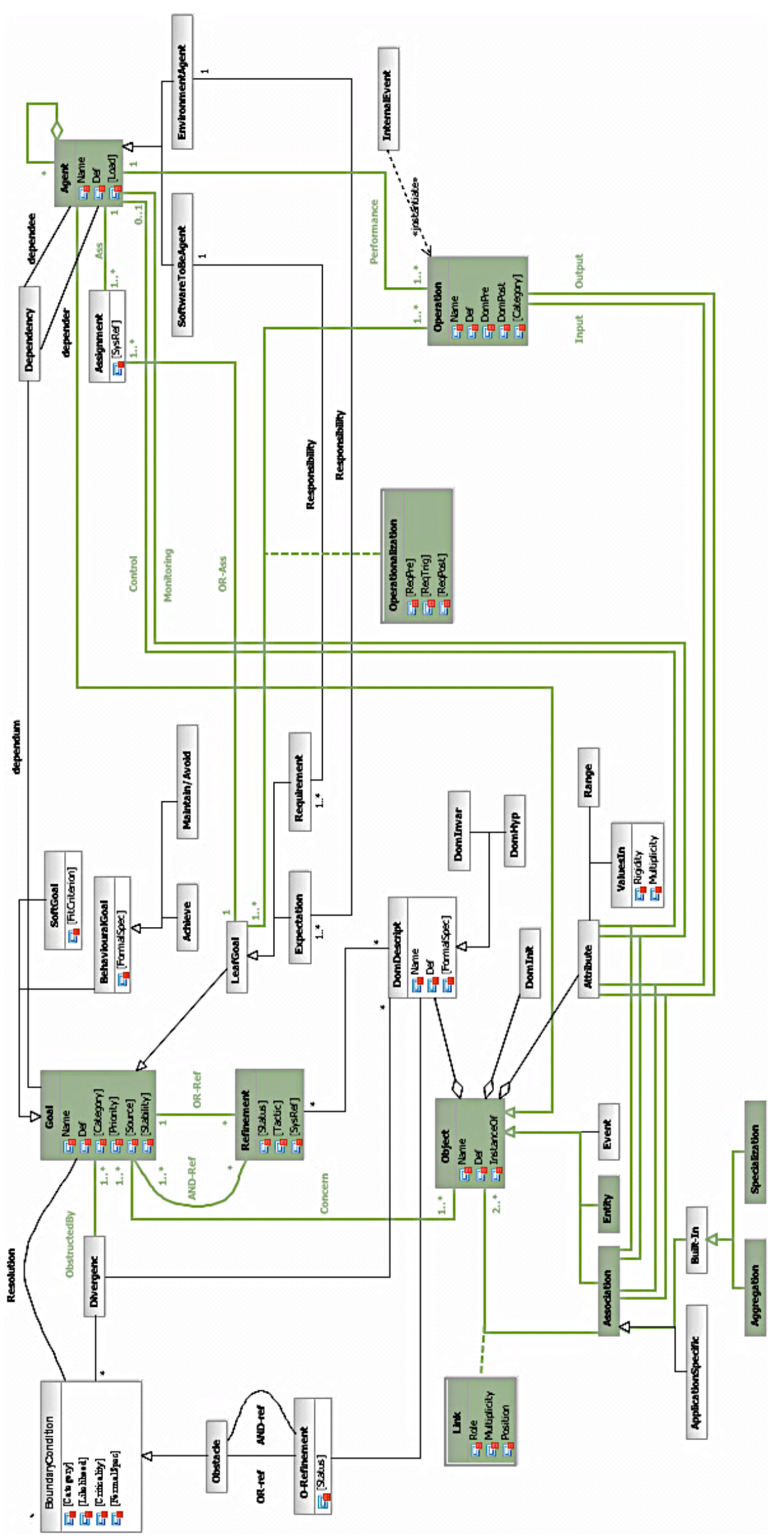

Fig. 12 KAOS elements being retained in CHOOSE 


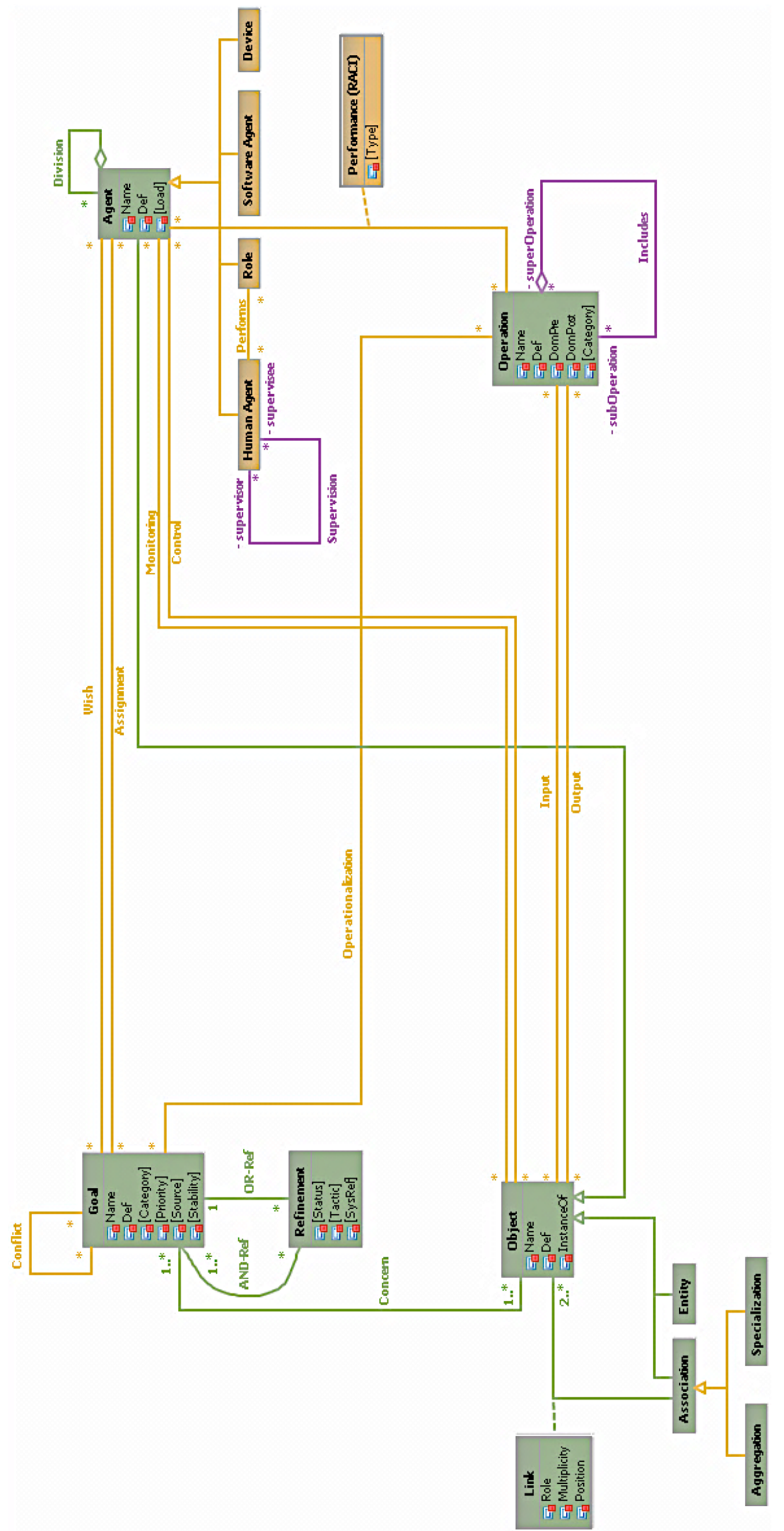

Fig. 13 Adjusting and adding elements from KAOS to CHOOSE 


\section{Appendix 2: OCL Constraints}

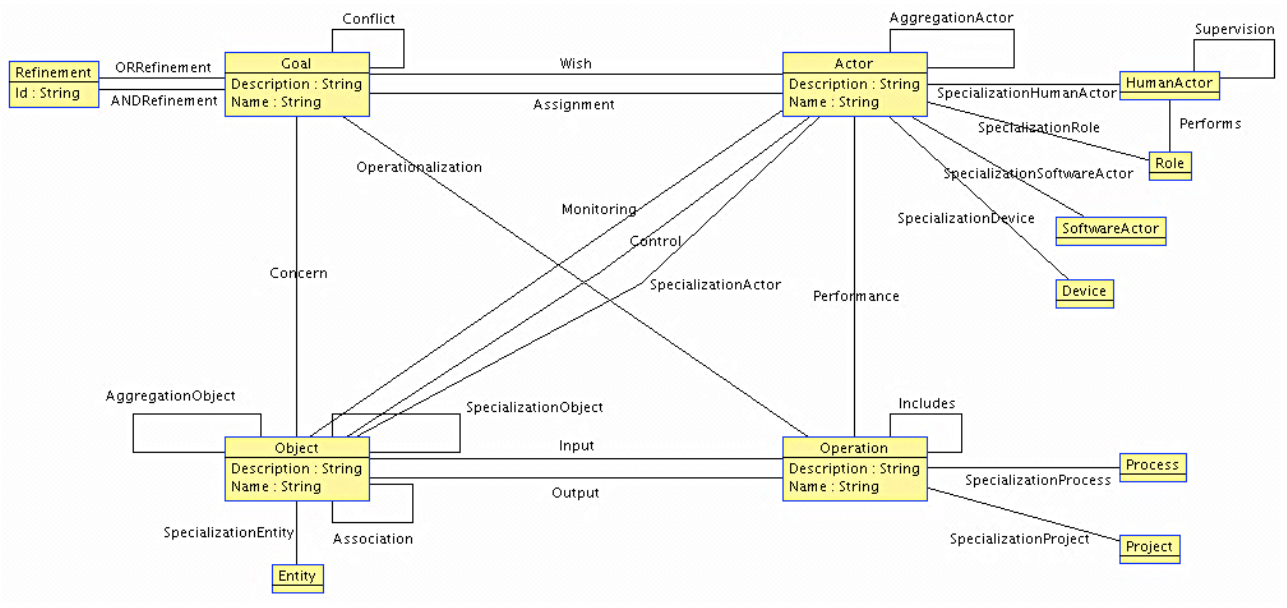

Fig. 14 CHOOSE metamodel in USE tool

The complete CHOOSE metamodel's classes and associations were input in the USE tool (Fig. 14). Next, constraints were added and tested by instantiating the metamodel in the tool. In Table 3 the metamodel including constraints is presented as a text file serving as an input for the USE tool. The objectified relationships Association, Aggregation, and Specialization are defined as normal relationships and the association class of Link and Performance is not shown. Due to tool limits, both aggregation and specialization relationships are modelled as normal associations. If interested, this text file can be used directly as an input for the USE tool following the guidelines on (Database Systems Group 2013) to test the metamodel and OCL constraints.

Table 3 CHOOSE metamodel and constraints as input for the USE tool

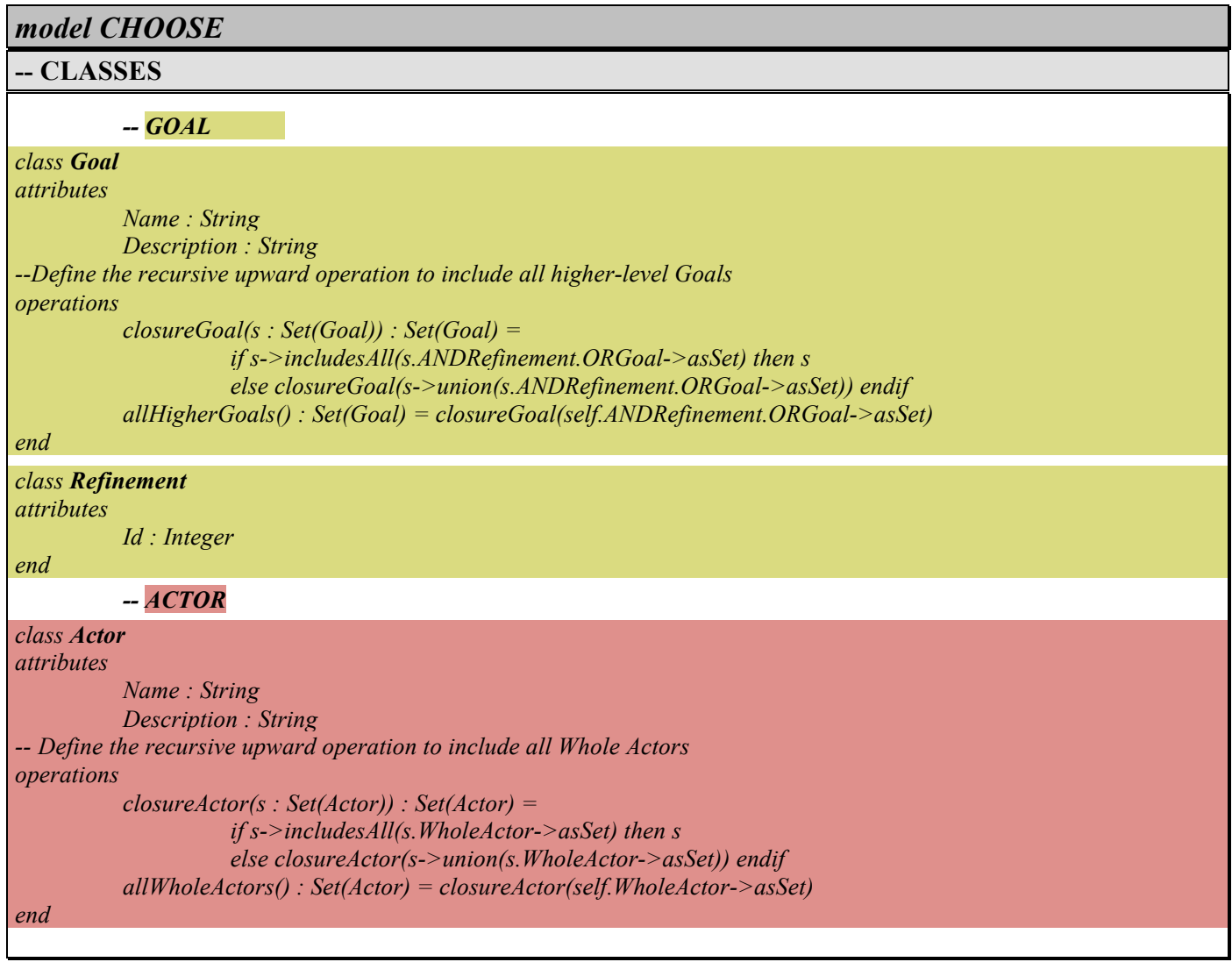




\section{class HumanActor}

-- Define the recursive upward operation to include all Supervisors

operations

closureHumanActor(s : Set(HumanActor)) : Set(HumanActor $)=$

if $s$->includesAll(s.Supervisor->asSet) then $s$

else closureHumanActor(s-> union(s.Supervisor->asSet)) endif

allSupervisors() : Set(HumanActor) = closureHumanActor(self.Supervisor->asSet)

end

class Role

end

class SoftwareActor

end

class Device

end

-- OPERATION

\section{class Operation}

attributes

Name : String

Description : String

-- Define the recursive upward operation to include all SuperOperations

operations

closureOperation $(s: \operatorname{Set}($ Operation $)):$ Set(Operation $)=$

if $s$->includesAll(s.SuperOperation->asSet) then $s$

else closureOperation(s-> union(s.SuperOperation->asSet)) endif

allSuperOperations() : Set(Operation) = closureOperation(self.SuperOperation->asSet)

end

\section{class Process}

end

class Project

end

\section{-- OBJECT}

\section{class Object}

attributes

Name : String

Description : String

-- Define the recursive upward operation to include all upper SuperObjects

operations

closureObject $(s: \operatorname{Set}($ Object $)): \operatorname{Set}($ Object $)=$

if $s$->includesAll(s.SuperObject->asSet) then $s$

else closureObject(s-> union(s.SuperObject->asSet)) endif

allSuperObjects() : Set(Object) $=$ closureObject(self.SuperObject $->$ asSet $)$

end

class Entity

end

\section{-- ASSOCIATIONS}

- $G O A L$

association ORRefinement between

Goal[1..1] role ORGoal

Refinement[*] role ORRefinement

end

association ANDRefinement between

Goal[1.. *] role ANDGoal

Refinement [*] role ANDRefinement

end

association Conflict between

Goal[*] role ConflictGoal1

Goal [*] role ConflictGoal2

end

\section{-- GOAL-ACTOR}

association Wish between

Goal [*] role WishGoal

Actor $\left.{ }^{*}\right]$ role WishActor

end

association Assignment between

Goal[*] role AssignmentGoal

Actor [*] role AssignmentActor 


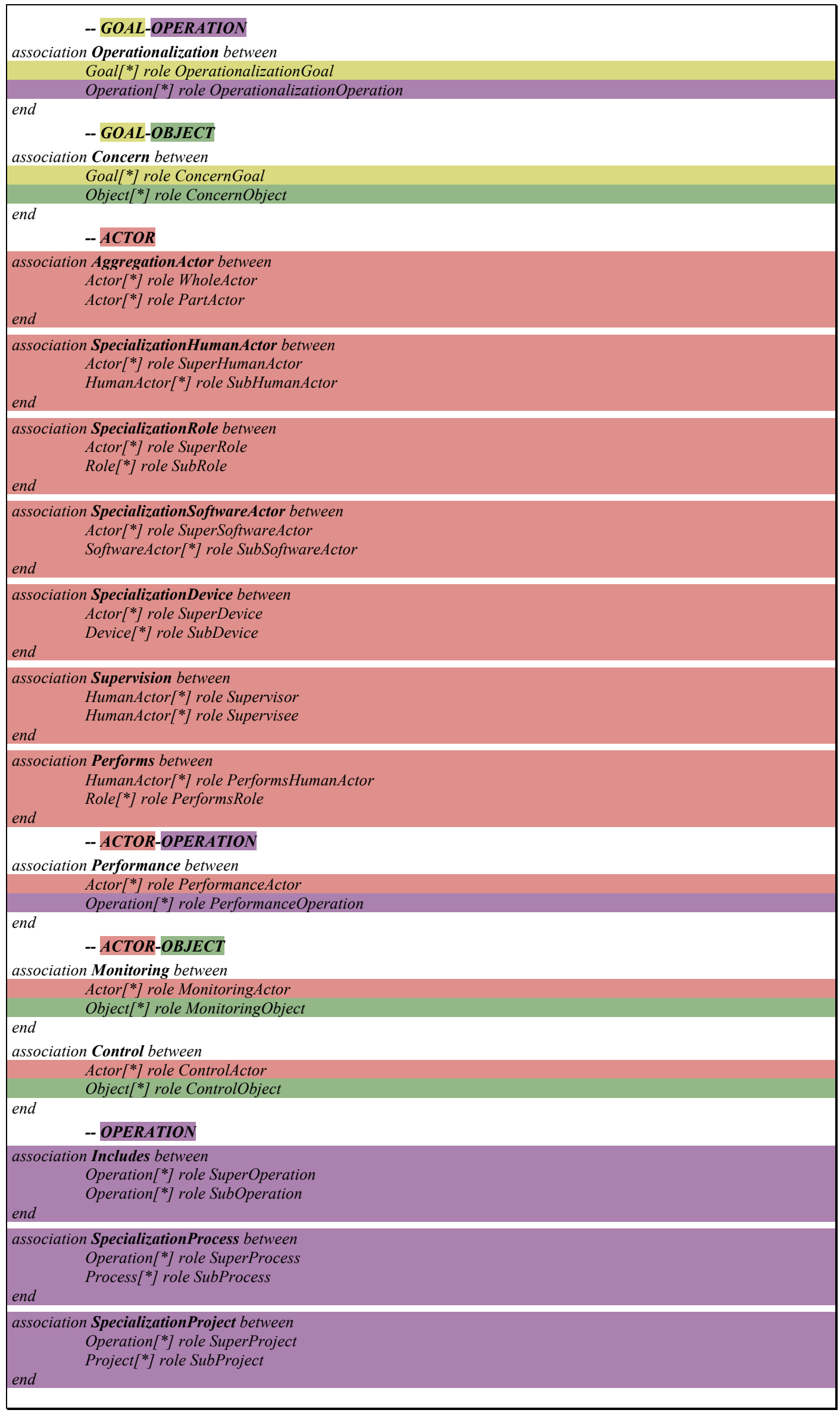




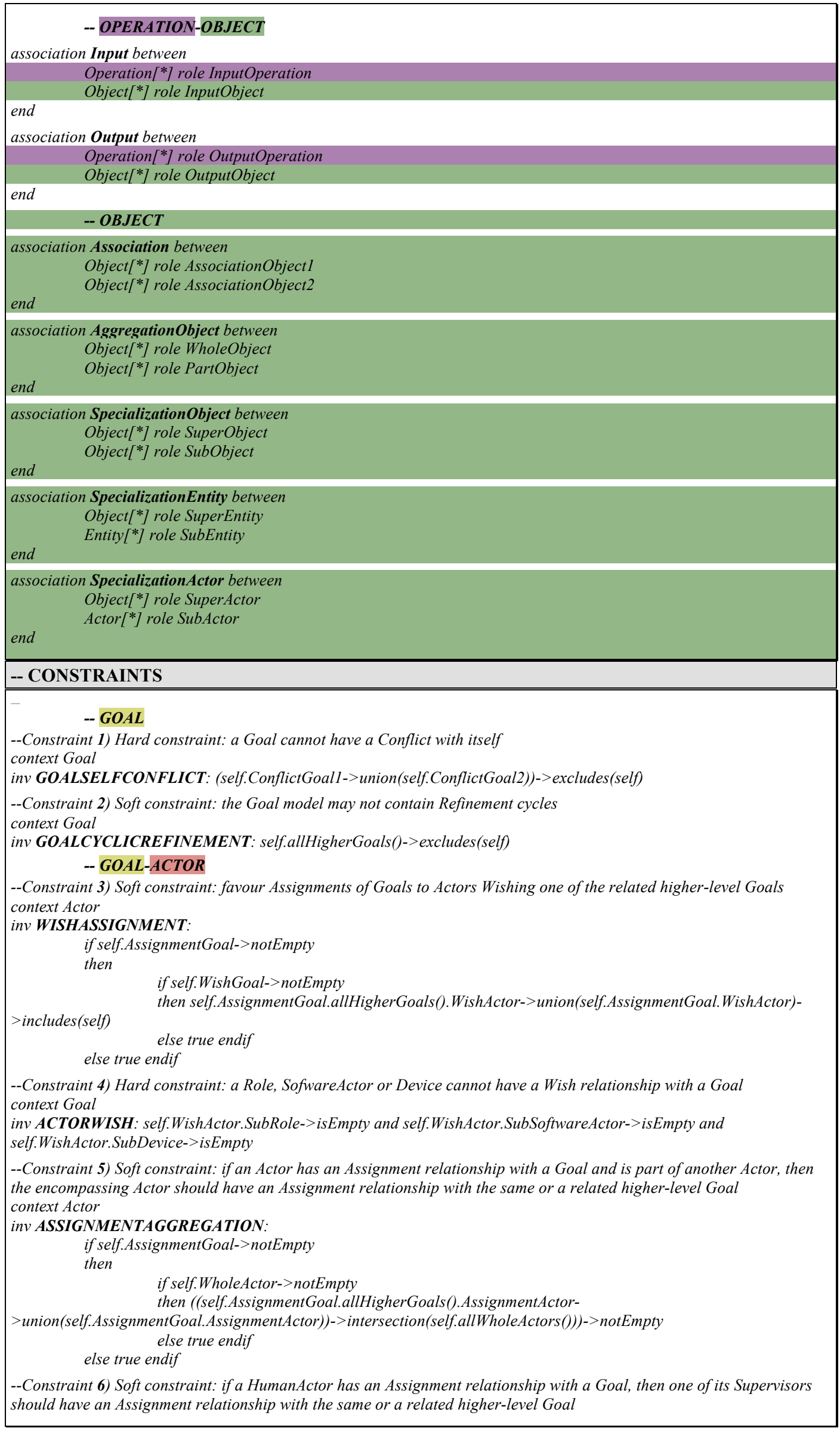




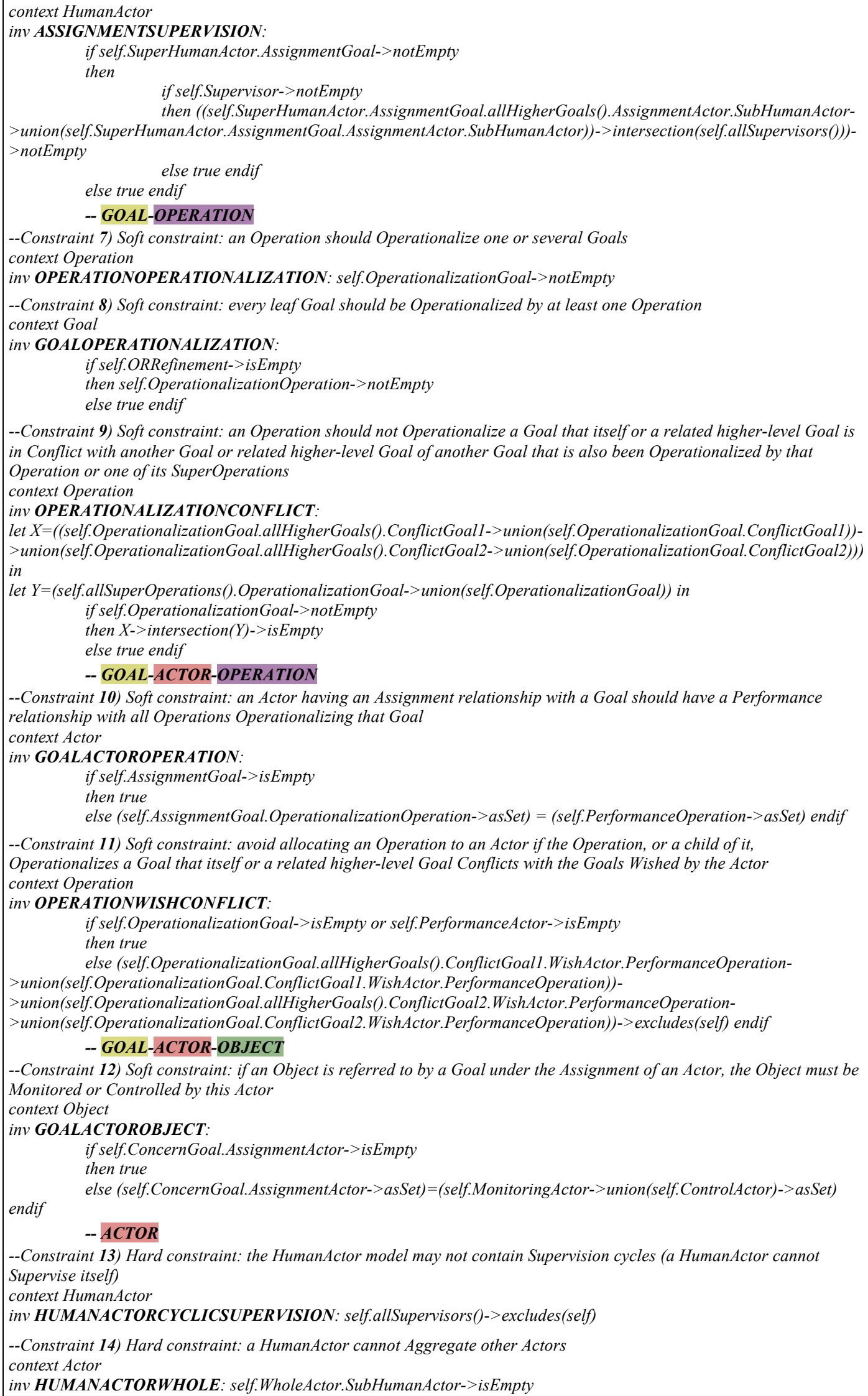

--Constraint 10) Soft constraint: an Actor having an Assignment relationship with a Goal should have a Performance relationship with all Operations Operationalizing that Goal 
--Constraint 15) Hard constraint: an Actor can only be Aggregated by other unspecialized Actors context Actor

inv ACTORAGGREGATION:

if self.SubHumanActor->isEmpty and self.SubRole->isEmpty and self.SubSoftwareActor->isEmpty and

self.SubDevice->isEmpty

then self.WholeActor.SubHumanActor->isEmpty and self.WholeActor.SubRole->isEmpty and

self.WholeActor.SubSoftwareActor->isEmpty and self.WholeActor.SubDevice->isEmpty else true endif

--Constraint 16) Hard constraint: a HumanActor can only be Aggregated by unspecialized Actors

context HumanActor

inv HUMANACTORAGGREGATION: self.SuperHumanActor. WholeActor. SubHumanActor->isEmpty and

self.SuperHumanActor. WholeActor.SubRole->isEmpty and self.SuperHumanActor. WholeActor.SubSoftwareActor-

$>$ isEmpty and self.SuperHumanActor.WholeActor.SubDevice->isEmpty

--Constraint 17) Hard constraint: a Role can only be Aggregated by other Roles or unspecialized Actors

context Role

inv ROLEAGGREGATION: self.SuperRole. WholeActor.SubHumanActor->isEmpty and

self.SuperRole. WholeActor.SubSoftwareActor->isEmpty and self.SuperRole. WholeActor.SubDevice->isEmpty

--Constraint 18) Hard constraint: a SoftwareActor can only be Aggregated by other SoftwareActors or unspecialized Actors

context SoftwareActor

inv SOFTWAREACTORAGGREGATION: self. SuperSoftwareActor. WholeActor. SubHumanActor->isEmpty and self.SuperSoftwareActor. WholeActor.SubRole->isEmpty and self.SuperSoftwareActor. WholeActor.SubDevice->isEmpty

--Constraint 19) Hard constraint: a Device can only be Aggregated by other Devices or unspecialized Actors context Device

inv DEVICEAGGREGATION: self.SuperDevice. WholeActor.SubHumanActor->isEmpty and

self.SuperDevice.WholeActor.SubRole->isEmpty and self.SuperDevice.WholeActor.SubSoftwareActor->isEmpty

--Constraint 20) Hard constraint: the Actor model may not contain Aggregation cycles (an Actor cannot contain itself)

context Actor

inv ACTORCYCLICAGGREGATION: self.allWholeActors()->excludes(self)

\section{-- ACTOR-OPERATION}

--Constraint 21) Soft constraint: every Operation should be under the Performance of at least one Actor

context Operation

inv OPERATIONPERFORMANCE: self.PerformanceActor->notEmpty

--Constraint 22) Soft constraint: if an Actor has a Performance relationship with an Operation and is part of one or more Actors, then at least one of those other Actors should have a Performance relationship with the same Operation, or one of its SuperOperations

context Actor

inv PERFORMANCEAGGREGATION

if self.PerformanceOperation->notEmpty

then

if self. WholeActor->notEmpty

then ((self.PerformanceOperation.allSuperOperations().PerformanceActor-

$>$ union(self.PerformanceOperation.PerformanceActor))->intersection(self.allWholeActors()))->notEmpty

else true endif else true endif

\section{-- ACTOR-OPERATION-OBJECT}

--Constraint 23) Soft constraint: the Inputs of an Operation Performed by an Actor should be Monitored by the Actor context Actor

inv ACTOROPERATIONOBJECTINPUT

if self.PerformanceOperation.InputObject->isEmpty

then true

else (self.MonitoringObject->intersection(self.PerformanceOperation.InputObject)-

$>$ as Set $)=($ self.PerformanceOperation.InputObject $->$ as Set $)$ endif

--Constraint 24) Soft constraint: the Outputs of an Operation Performed by an Actor should be Controlled by the Actor context Actor

inv ACTOROPERATIONOBJECTOUTPUT:

if self.PerformanceOperation.OutputObject->isEmpty

then true

else (self.ControlObject->intersection(self.PerformanceOperation.OutputObject)-

$>$ as Set $)=($ self.PerformanceOperation.OutputObject $->$ as Set $)$ endif

-- OPERATION

--Constraint 25) Hard constraint: the Operation model may not contain Includes cycles (Operation cannot Include itself)

context Operation

inv OPERATIONCYCLICINCLUDES: self.allSuperOperations()->excludes(self)

-- OBJECT

--Constraint 26) Hard constraint: the Object model may not contain Specialization cycles (Object cannot Specialize itself)

context Object

inv OBJECTCYCLICSPECIALIZATION: self.allSuperObjects()->excludes(self) 